Chapter 22

\title{
Photorefractive Effect in Ferroelectric Liquid Crystals
}

\author{
Takeo Sasaki \\ Additional information is available at the end of the chapter \\ http://dx.doi.org/10.5772/52921
}

\section{Introduction}

Ferroelectric liquid crystals have been attracting great interest for their application in photorefractive devices. The photorefractive effect is a phenomenon by which a change in refractive index is induced by the interference of laser beams [1,2]. Dynamic holograms are easily realized by the photorefractive effect. Holograms generate three-dimensional images of objects. They are produced by recording interference fringes generated by light reflected from an object and a reference light (Figure 1). A hologram diffracts incident light to produce a three-dimensional images of an object. 3D displays are expected to be widely used as nextgeneration displays. However, current 3D displays are essentially stereograms. However, holographic displays that can realize natural 3D images are anticipated.

The photorefractive effect has the potential to realize dynamic holograms by recording holograms as a change in the refractive index of the medium [1,2]. The photorefractive effect induces a change in the refractive index by a mechanism involving both photovoltaic and electro-optic effects (Figure 2). When two laser beams interfere in an organic photorefractive material, a charge generation occurs at the bright positions of the interference fringes. The generated charges diffuse or drift within the material. Since the mobilities of positive and negative charges are different in most organic materials, a charge separated state is formed. The charge with the higher mobility diffuses over a longer distance than the charge with lower mobility, such that while the low mobility charge stays in the bright areas, the high mobility charge moves to the dark areas. The bright and dark positions of the interference fringes are thus charged with opposite polarities and an internal electric field (space-charge field) is generated in the area between the bright and dark positions. The refractive index of this area between the bright and dark positions is changed through the electro-optic effect. Thus, a refractive index grating (or hologram) is formed. One material class that exhibits high photorefractivity is that of glassy photoconductive polymers doped with high concentrations of D- $\pi$-A chromophores, in which donor and acceptor groups are attached to a $\pi$ - 
conjugate system [3-5]. In order to obtain photorefractivity in polymer materials, a high electric field of $10-50 \mathrm{~V} / \mu \mathrm{m}$ is usually applied to a polymer film [6-8]. This electric field is necessary to increase charge generation efficiency. Significantly, the photorefractive effect permits two-beam coupling. It can be used to coherently amplify signal beams, and so has the potential to be used in a wide range of optical technologies as a transistor in electric circuits. With the aim of developing a 3D display, a multiplex hologram has been demonstrated using a photorefractive polymer film $[9,10]$. Clear 3D images were recorded in the film. However, the slow response ( 100 ms) and the high electric field $(30-50 \mathrm{~V} / \mu \mathrm{m})$ required to activate the photorefractive effect in the polymer materials both need to be improved.

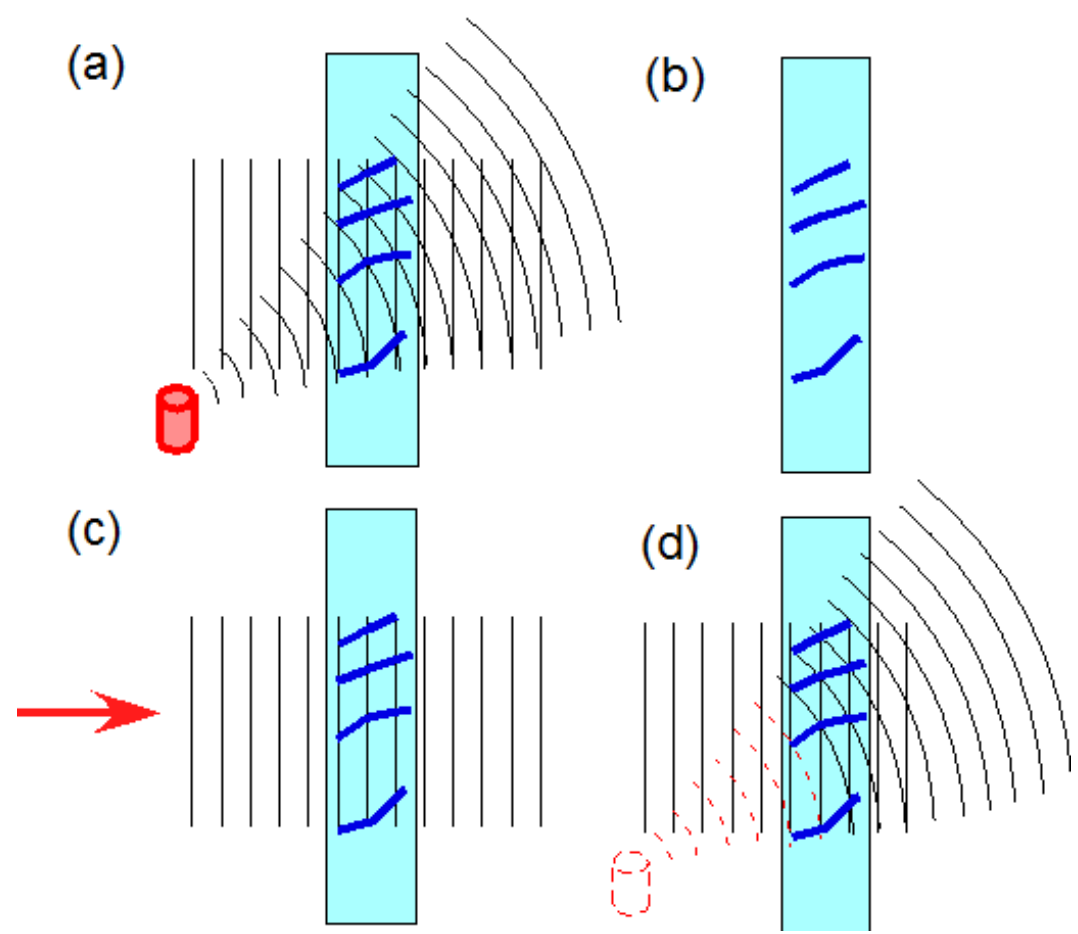

Figure 1. Hologram recording and image reconstruction. (a) A light reflected from an object is interfered with by a reference light in a photo-sensitive material, such as a photopolymer. (b) The interference fringe is recorded in a photosensitive material (hologram). (c) A readout beam is irradiated by the hologram. (d) The readout beam is diffracted by the hologram and the object image is reconstructed.

Ferroelectric liquid crystals are expected to be used as high performance photorefractive materials [11-13]. A photorefractive ferroelectric liquid crystal with a fast response of $5 \mathrm{~ms}$ has been reported [14]. The photorefractive effect has been reported in surface-stabilized ferroelectric liquid crystals (SS-FLCs) doped with a photoconductive compound. Liquid crystals are classified into several groups. The most well known are nematic liquid crystals and smectic liquid crystals. 

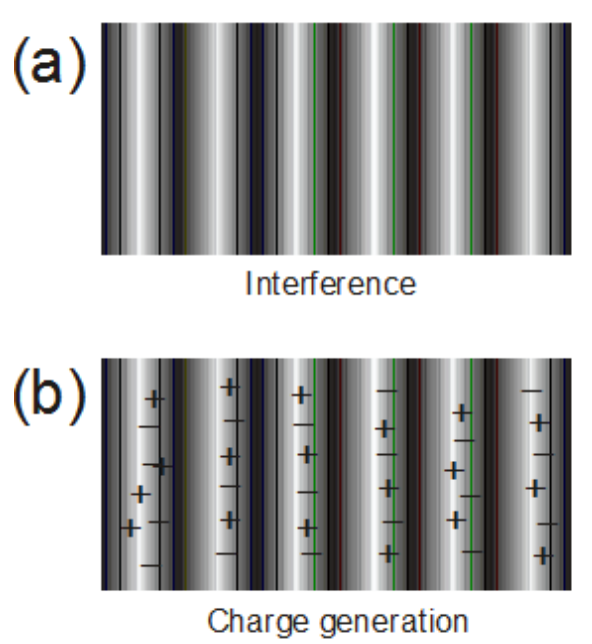
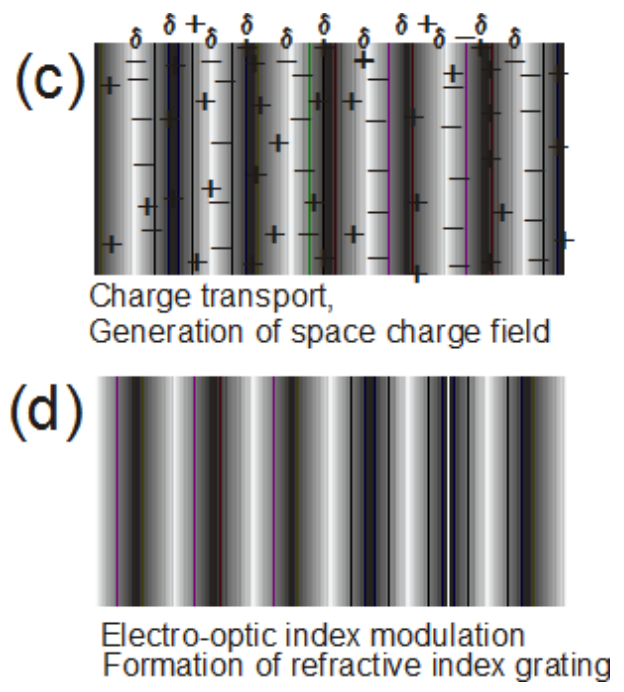

Figure 2. Schematic illustration of the mechanism of the photorefractive effect. (a) Two laser beams interfere in the photorefractive material; (b) charge generation occurs at the light areas of the interference fringes; (c) electrons are trapped at the trap sites in the light areas and holes migrate by diffusion or drift in the presence of an external electric field and generate an internal electric field between the light and dark positions; $(d)$ the refractive index of the corresponding area is altered by the internal electric field generated.

Nematic liquid crystals are used in LC displays. On the other hand, smectic liquid crystals are very viscous and, hence, are not utilized in any practical applications. Ferroelectric liquid crystals (FLCs) belong to the class of smectic liquid crystals that have a layered structure (Figure 3). The molecular structure of a typical FLC contains a chiral unit, a carbonyl group, a central core - which is a rigid rod-like structure, such as biphenyl, phenylpyrimidine or phenylbenzoate - and a flexible alkyl chain [15]. Thus, the dipole moment of an FLC molecule is perpendicular to the molecular long axis. FLCs exhibit a chiral smectic C phase $\left(\mathrm{SmC}^{*}\right)$ that possesses a helical structure. Compared to nematic LCs, FLCs are more crystal than liquid and the preparation of fine FLC films requires several sophisticated techniques. Obtaining a uniformly aligned, defect-free, surface-stabilized FLC (SS-FLC, Figure 3) using a single FLC compound is very difficult, and mixtures of several LC compounds are usually used to obtain fine SS-FLC films. 

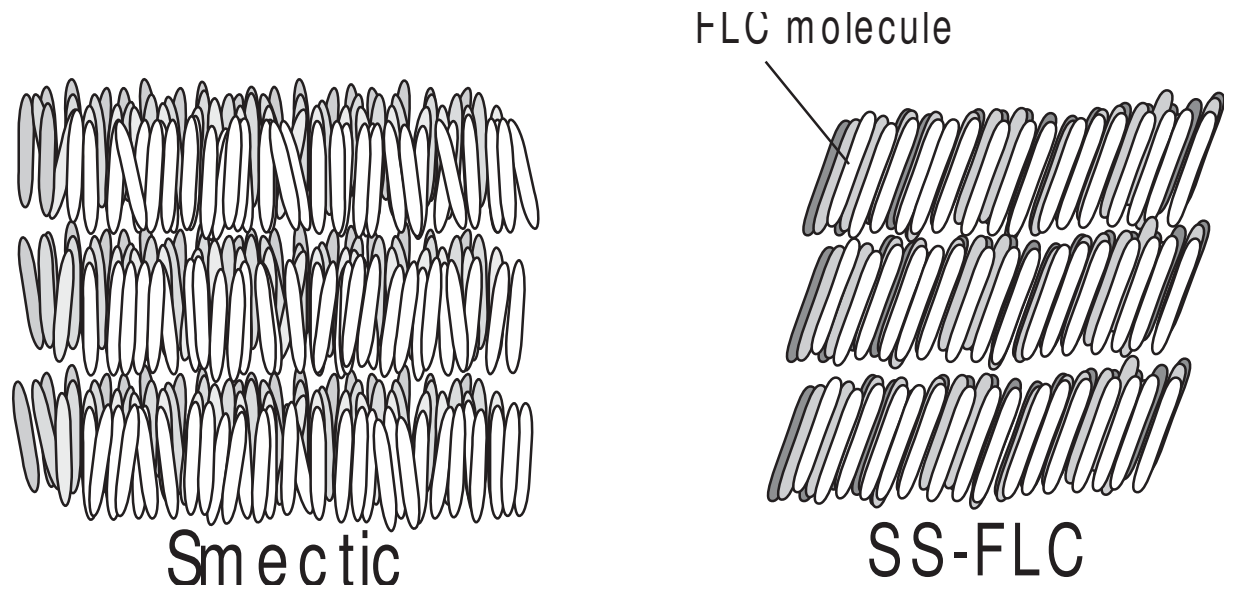

Figure 3. Structures of the smectic phase and the surface stabilized smectic-C phase (SS-FLC).

The FLC mixtures are composed of the base LC - which is also a mixture of several LC compounds - and a chiral compound. The chiral compound introduces a helical structure into the LC phase through supramolecular interactions. It should be mentioned here that in order to observe ferroelectricity in these materials, the ferroelectric liquid crystals must be formed into thin films. The thickness of the film must be within a few micrometers. When an FLC is sandwiched between glass plates to form a film a few micrometers thick, the helical structure of the smectic C phase uncoils and a surface-stabilized state (SS-state) is formed in which spontaneous polarization (Ps) appears. For display applications, the thickness of the film is usually $2 \mu \mathrm{m}$. In such thin films, FLC molecules can align in only two directions. This state is called a surface-stabilized state (SS-state). The alignment direction of the FLC molecules changes according to the direction of the spontaneous polarization (Figure 4). The direction of the spontaneous polarization is governed by the photoinduced internal electric field, giving rise to a refractive index grating with properties dependent on the direction of polarization.

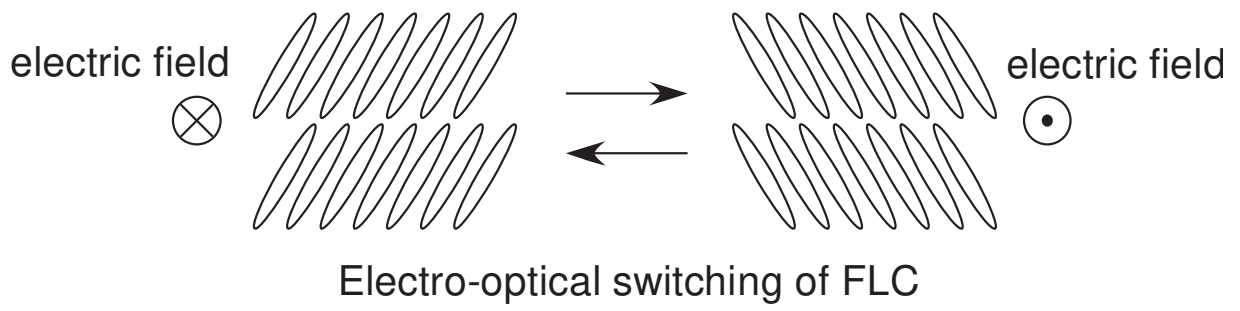

Figure 4. Electro-optical switching in the surface-stabilized state of FLCs. 
Figure 5 shows a schematic illustration of the mechanism of the photorefractive effect in FLCs. When laser beams interfere in a mixture of an FLC and a photoconductive compound, charge separation occurs between bright and dark positions and an internal electric field is produced. The internal electric field alters the direction of spontaneous polarization in the area between the bright and dark positions of the interference fringes, which induces a periodic change in the orientation of the FLC molecules. This is different from the processes that occur in other photorefractive materials in that the molecular dipole rather than the bulk polarization responds to the internal electric field. Since the switching of FLC molecules is due to the response of bulk polarization, the switching is extremely fast.
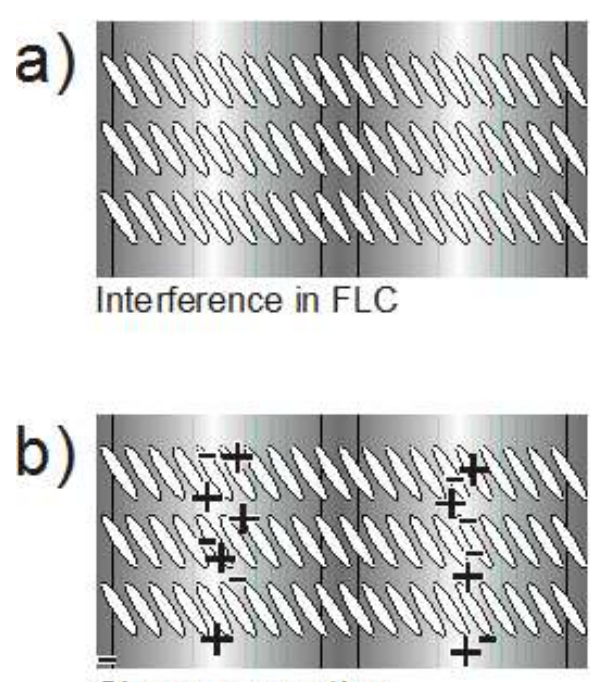

Charge generation
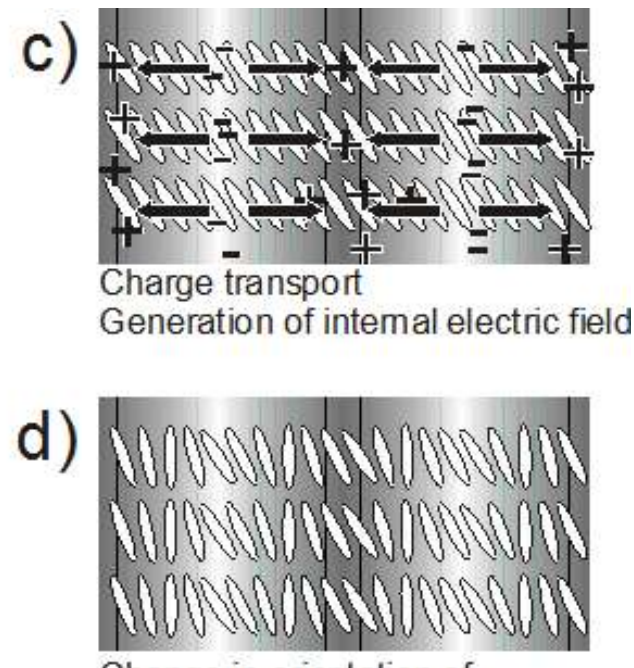

Change in orientation of FLC molecules

Figure 5. Schematic illustration of the mechanism of the photorefractive effect in FLCs. (a) Two laser beams interfere in the surface-stabilized state of the FLC/photoconductive compound mixture; (b) charge generation occurs at the bright areas of the interference fringes; (c) electrons are trapped at the trap sites in the bright areas, holes migrate by diffusion or drift in the presence of an external electric field to generate an internal electric field between the bright and dark positions; (d) the orientation of the spontaneous polarization vector (i.e., the orientation of mesogens in the FLCs) is altered by the internal electric field.

\section{Characteristics of the photorefractive effect}

Since a change in the refractive index via the photorefractive effect occurs in the areas between the bright and dark positions of the interference fringe, the phase of the resulting index grating is shifted from the interference fringe. This is characteristic of the photorefractive effect that the phase of the refractive index grating is $\pi / 2$-shifted from the interference fringe. When the material is photochemically active and is not photorefractive, 
a photochemical reaction takes place at the bright areas and a refractive index grating with the same phase as that of the interference fringe is formed (Figure 6(a)).

(a) Photochromic

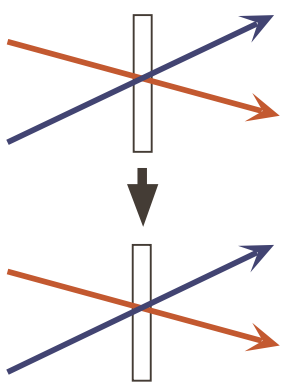

(b) Photorefractive

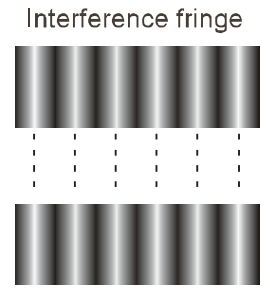

Refractive index grating

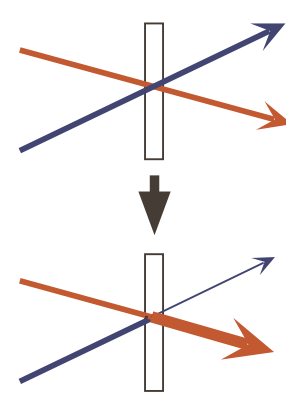

Asymmetric Energy Exchange
Interference fringe

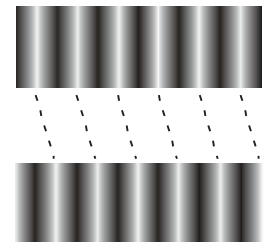

Refractive index grating

Figure 6. (a) Photochromic grating, and (b) photorefractive grating.

The interfering laser beams are diffracted by this grating; however, the apparent transmitted intensities of the laser beams do not change because the diffraction is symmetric. Beam 1 is diffracted in the direction of beam 2 and beam 2 is diffracted in the direction of beam 1 . However, if the material is photorefractive, the phase of the refractive index grating is shifted from that of the interference fringes, and this affects the propagation of the two beams. Beam 1 is energetically coupled with beam 2 for the two laser beams. Consequently, the apparent transmitted intensity of beam 1 increases and that of beam 2 decreases (Figure 6(b)). This phenomenon is termed 'asymmetric energy exchange' in the two-beam coupling experiment. The photorefractivity of a material is confirmed by the occurrence of this asymmetric energy exchange.

\section{Measurement of photorefractivity}

The photorefractive effect is evaluated by a two-beam coupling method and by a four-wave mixing experiment. Figure 7(a) shows a schematic illustration of the experimental setup used for the two-beam coupling method. A p-polarized beam from a laser is divided into two beams by a beam splitter and the beams are interfered within the sample film. An electric field is applied to the sample using a high voltage supply unit. This external electric field is applied in order to increase the efficiency of charge generation in the film. The change in the transmitted beam intensity is monitored. If a material is photorefractive, an asymmetric energy exchange is observed. The magnitude of photorefractivity is evaluated using a parameter called the gain coefficient, which is calculated from the change in the transmitted intensity of the laser beams induced through the two-beam coupling [1]. In order to calculate the two-beam coupling gain coefficient, it must be determined whether the 
diffraction condition is within the Bragg regime or within the Raman-Nath regime. These diffraction conditions are distinguished by a dimensionless parameter $\mathrm{Q}$.

$$
\mathrm{Q}=2 \pi \mathrm{lL} / \mathrm{n} \Lambda^{2}
$$

(a) FLC sample

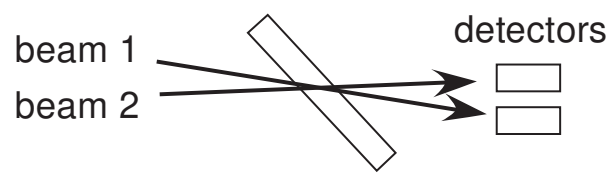

(b) FLC sample

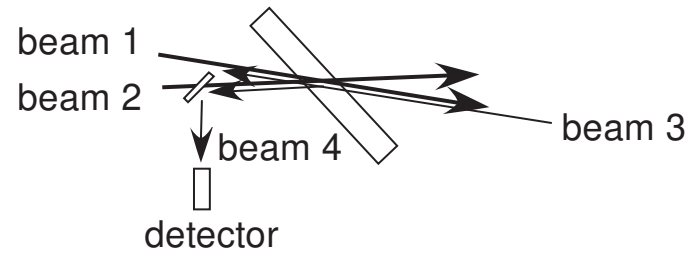

Figure 7. Schematic illustrations of the experimental set-up for the (a) two-beam coupling, and (b) four-wave-mixing techniques.

$\mathrm{Q}>1$ is defined as the Bragg regime of optical diffraction. In this regime, multiple scattering is not permitted, and only one order of diffraction is produced. Conversely, $Q<1$ is defined as the Raman-Nath regime of optical diffraction. In this regime, many orders of diffraction can be observed. Usually, $Q>10$ is required to guarantee that the diffraction is entirely within the Bragg regime. When the diffraction is in the Bragg diffraction regime, the two-beam coupling gain coefficient $\Gamma\left(\mathrm{cm}^{-1}\right)$ is calculated according to the following equation:

$$
\Gamma=\frac{1}{D} \ln \left(\frac{g m}{1+m-g}\right)
$$

where $D=L / \cos (\theta)$ is the interaction path for the signal beam ( $L=$ sample thickness, $\theta=$ propagation angle of the signal beam in the sample), $g$ is the ratio of the intensities of the signal beam behind the sample with and without a pump beam, and $\mathrm{m}$ is the ratio of the beam intensities (pump/signal) in front of the sample.

A schematic illustration of the experimental setup used for the four-wave mixing experiment is shown in Figure 7(b). S-polarized writing beams are interfered in the sample film and the diffraction of a p-polarized probe beam, counter-propagating to one of the writing beams, is measured. The diffracted beam intensity is typically measured as a function of time, applied (external) electric field and writing beam intensities, etc. The diffraction efficiency is defined as the ratio of the intensity of the diffracted beam and the intensity of the probe beam that is transmitted when no grating is present in the sample due to the writing beams. In probing the grating, it is important that beam 3 does not affect the grating or interact with the writing beams. This can be ensured by making the probe beam much weaker than the writing beams and by having the probe beam polarized orthogonal to the writing beams. 


\section{Photorefractive effect of FLCs}

\subsection{Two-beam coupling experiments on FLCs}

The photorefractive effect in an FLC was first reported by Wasielewsky et al. in 2000 [11]. Since then, details of photorefractivity in FLC materials have been further investigated by Sasaki et al. and Golemme et al. [12-14]. The photorefractive effect in a mixture of an FLC and a photoconductive compound was measured in a two-beam coupling experiment using a $488 \mathrm{~nm} \mathrm{Ar}$ laser. The structures of the photoconductive compounds used are shown in Figure 8. A commercially available FLC, SCE8 (Clariant), was used. CDH was used as a photoconductive compound and TNF was used as a sensitizer. The concentrations of CDH and TNF were $2 \mathrm{wt} \%$ and $0.1 \mathrm{wt} \%$ respectively. The samples were injected into a 10- $\mu \mathrm{m}$-gap glass cell equipped with $1 \mathrm{~cm}^{2}$ ITO electrodes and a polyimide alignment layer (Figure 9).
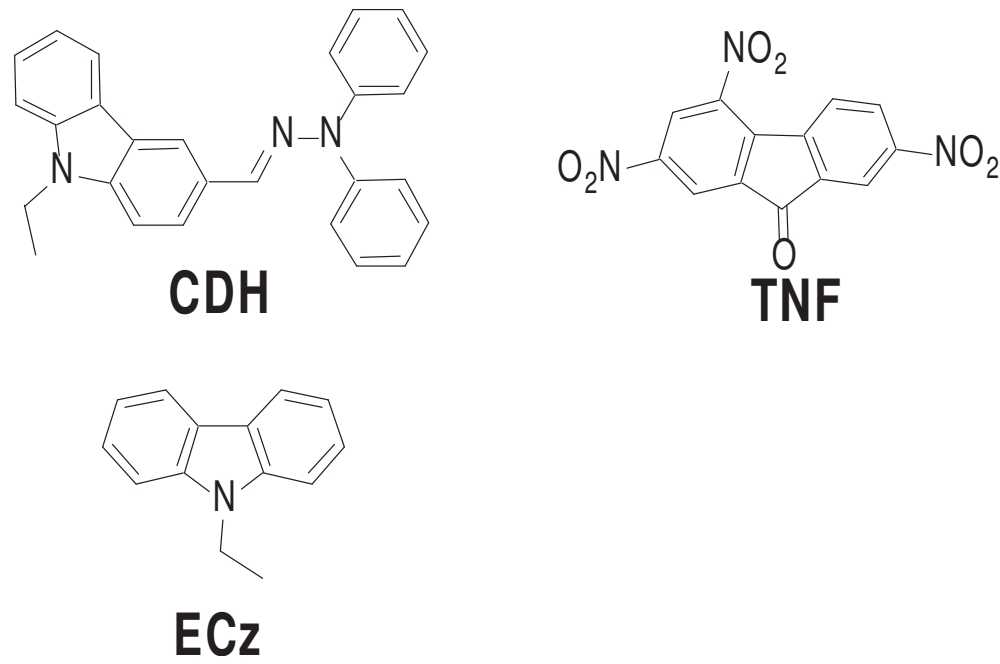

Figure 8. Structures of the photoconductive compound $\mathrm{CDH}, \mathrm{ECz}$ and the sensitizer TNF

Incident beams $(488 \mathrm{~nm})$

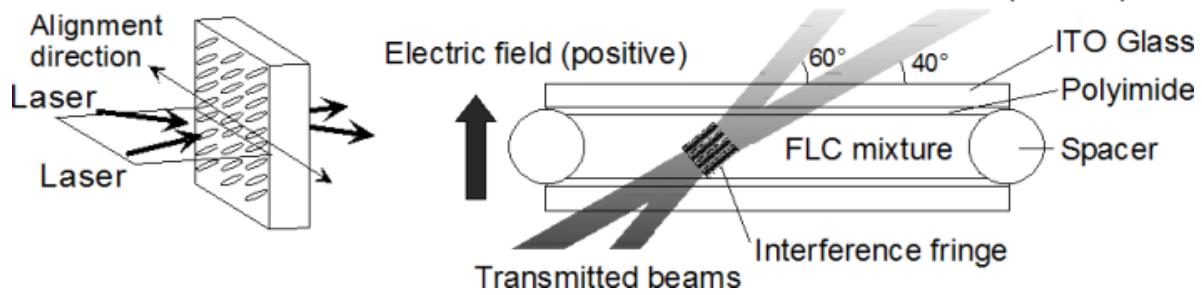

Figure 9. Laser beam incidence condition and the structure of the LC cell. 
Figure 10 shows a typical example of asymmetric energy exchange observed in the FLC(SCE8)/CDH/TNF sample under an applied DC electric field of $0.1 \mathrm{~V} / \mu \mathrm{m}$ [12]. The interference of the divided beams in the sample resulted in the increased transmittance of one beam and the decreased transmittance of the other. The change in the transmitted intensities of the two beams is completely symmetric, as can be seen in Figure 10. This indicates that the phase of the refractive index grating is shifted from that of the interference fringes. The grating formation was within the Bragg diffraction regime and no higher-order diffraction was observed under the conditions used.

The temperature dependence of the gain coefficient of SCE8 doped with $2 \mathrm{wt} \% \mathrm{CDH}$ and 0.1 wt $\%$ TNF is shown in Figure 11(a). Asymmetric energy exchange was observed only at temperatures below $46^{\circ} \mathrm{C}$. The spontaneous polarization of the identical sample is plotted as a function of temperature in Figure 11(b).

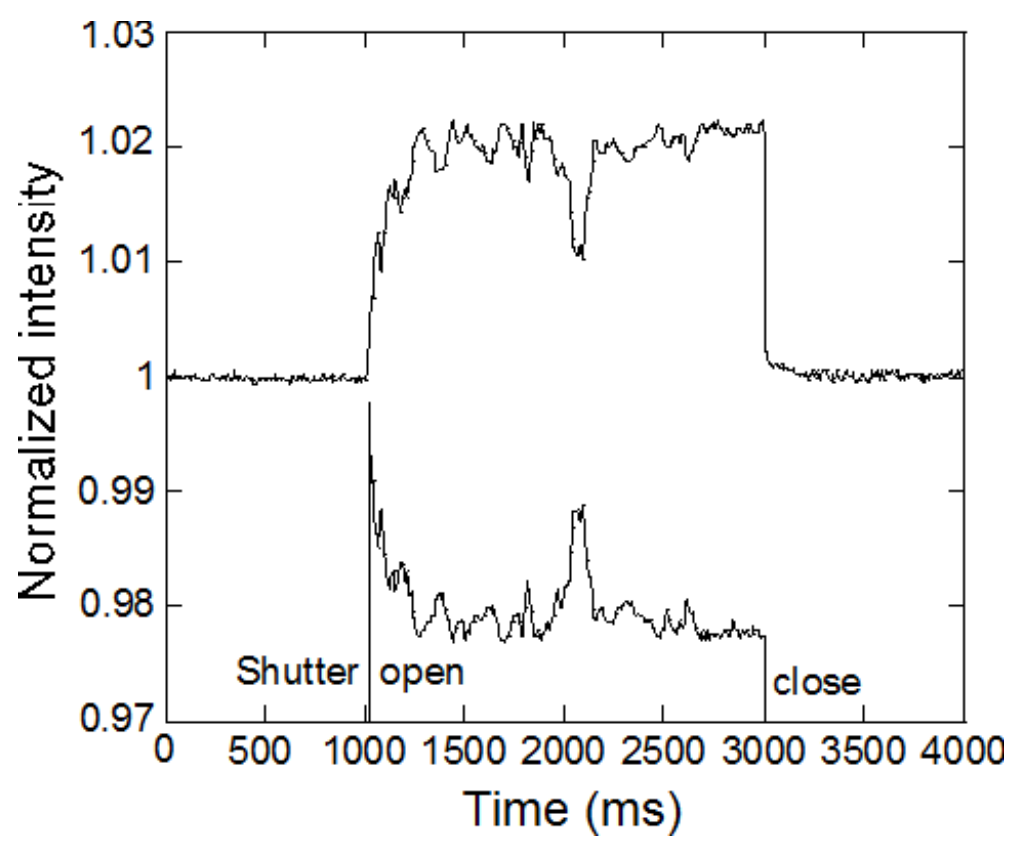

Figure 10. A typical example of asymmetric energy exchange observed in an FLC (SCE8) mixed with 2 wt\% CDH and $0.1 \mathrm{wt} \%$ TNF. An electric field of $+0.3 \mathrm{~V} / \mu \mathrm{m}$ was applied to the sample. 

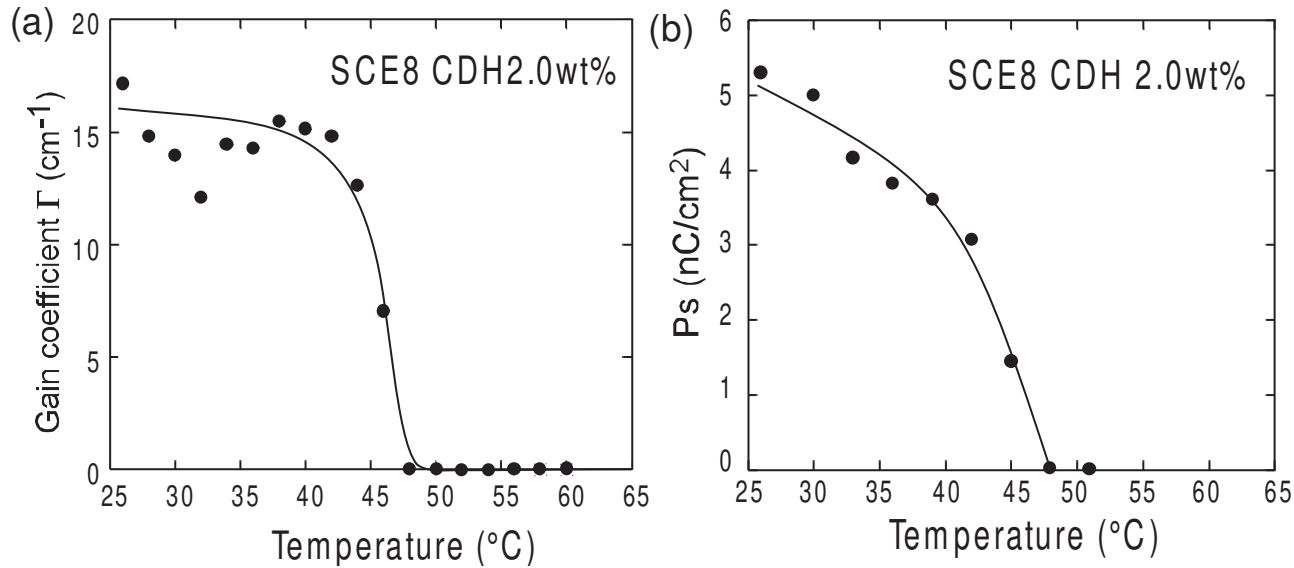

Figure 11. Temperature dependence of (a) the gain coefficient, and (b) the spontaneous polarization of an FLC (SCE8) mixed with 2 wt \% CDH and 0.1 wt\% TNF. For two-beam coupling experiments, an electric field of $0.1 \mathrm{~V} / \mu \mathrm{m}$ was applied to the sample.

Similarly, the spontaneous polarization vanished when the temperature was raised above $46^{\circ} \mathrm{C}$. Thus, asymmetric energy exchange was observed only in the temperature range in which the sample exhibited ferroelectric properties; in other words, the $\mathrm{SmC}^{*}$ phase. Since the molecular dipole moment of the FLCs is small and the dipole moment is aligned perpendicular to the molecular axis, large changes in the orientation of the molecular axis cannot be induced by an internal electric field in the SmA or $\mathrm{N}^{*}$ phase of the FLCs. However, in the $\mathrm{SmC}^{*}$ phase, reorientation associated with spontaneous polarization occurs due to the internal electric field. The spontaneous polarization also causes the orientation of FLC molecules in the corresponding area to change accordingly. A maximum resolution of $0.8 \mu \mathrm{m}$ was obtained in this sample.

\subsection{Effect of the magnitude of the applied electric field}

In polymeric photorefractive materials, the strength of the externally applied electric field is a very important factor. The external electric field is necessary to increase the charge separation efficiency sufficiently to induce a photorefractive effect. In other words, the photorefractivity of the polymer is obtained only with the application of a few $\mathrm{V} / \mu \mathrm{m}$ electric fields. The thickness of the polymeric photorefractive material commonly reported is about $100 \mu \mathrm{m}$, so the voltage necessary to induce the photorefractive effect is a few $\mathrm{kV}$. On the other hand, the photorefractive effect in FLCs can be induced by applying a very weak external electric field. The maximum gain coefficient for the FLC (SCE8) sample was obtained using an electric field strength of only $0.2-0.4 \mathrm{~V} / \mu \mathrm{m}$. The thickness of the FLC sample is typically $10 \mu \mathrm{m}$, so that the voltage necessary to induce the photorefractive effect is only a few V. The dependence of the gain coefficient of a mixture of FLC (SCE8)/CDH/TNF on the strength of the electric field is shown in Figure 12. The gain coefficient of SCE8 doped with $0.5-1 \mathrm{wt} \% \mathrm{CDH}$ increased with the strength of the external electric field. However, the gain coefficient of 
SCE8 doped with $2 \mathrm{wt} \% \mathrm{CDH}$ decreased when the external electric field exceeded $0.4 \mathrm{~V} / \mu \mathrm{m}$. The same tendency was observed for M4851/050 as well. The formation of an orientational grating is enhanced when the external electric field is increased from 0 to $0.2 \mathrm{~V} / \mu \mathrm{m}$ as a result of induced charge separation under a higher external electric field. However, when the external electric field exceeded $0.2 \mathrm{~V} / \mu \mathrm{m}$, a number of zigzag defects appeared in the surface-stabilized state. These defects cause light scattering and result in a decrease in the gain coefficient.

(a)

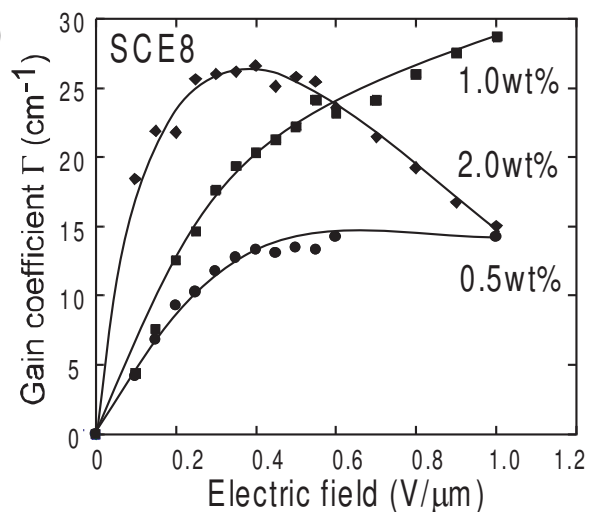

(b)

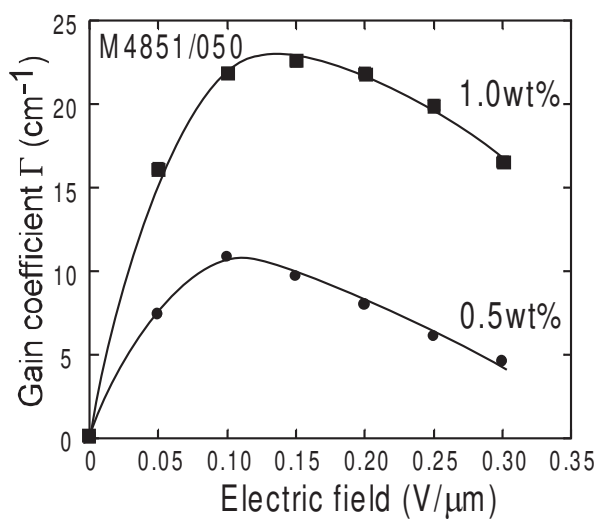

Figure 12. Electric field dependence of the gain coefficient of SCE8 and M4851/050 mixed with several concentrations of $\mathrm{CDH}$ and $0.1 \mathrm{wt} \% \mathrm{TNF}$ in a $10 \mu \mathrm{m}$-gap cell measured at $30^{\circ} \mathrm{C}$.

\subsection{Refractive index grating formation time}

The formation of a refractive index grating involves charge separation and reorientation. The index grating formation time is affected by these two processes and both may be ratedetermining steps. The refractive index grating formation times in SCE8 and M4851/050 were determined based on the simplest single-carrier model of photorefractivity $[1,2]$, wherein the gain transient is exponential. The rising signal of the diffracted beam was fitted using a single exponential function, shown in equation (3).

$$
\gamma(\mathrm{t})-1=(\gamma-1)[1-\exp (-\mathrm{t} / \tau)]^{2}
$$

Here, $\gamma(\mathrm{t})$ represents the transmitted beam intensity at time $\mathrm{t}$ divided by the initial intensity $(\gamma(\mathrm{t})=\mathrm{I}(\mathrm{t}) / \mathrm{I} 0)$ and $\tau$ is the formation time. The grating formation time in SCE8/CDH/TNF is plotted as a function of the strength of the external electric field in Figure 13(a). The grating formation time decreased with increasing electric field strength due to the increased efficiency of charge generation. The formation time was shorter at higher temperatures, corresponding to a decrease in the viscosity of the FLC with increasing temperature. The formation time for SCE8 was found to be $20 \mathrm{~ms}$ at $30^{\circ} \mathrm{C}$. As shown in Figure 13(b), the for- 
mation time for M4851/050 was found to be independent of the magnitude of the external electric field, with a time of $80-90 \mathrm{~ms}$ for M4851/050 doped with $1 \mathrm{wt} \% \mathrm{CDH}$ and $0.1 \mathrm{wt} \%$ TNF. This is slower than for SCE8, although the spontaneous polarization of M4851/050 (-14 $\left.\mathrm{nC} / \mathrm{cm}^{2}\right)$ is larger than that of SCE8 $\left(-4.5 \mathrm{nC} / \mathrm{cm}^{2}\right)$ and the response time of the electro-optical switching (the flipping of spontaneous polarization) to an electric field ( $\pm 10 \mathrm{~V}$ in a $2 \mu \mathrm{m}$ cell) is shorter for M4851/050. The slower formation of the refractive index grating in M4851/050 is likely due to the poor homogeneity of the SS-state and charge mobility.
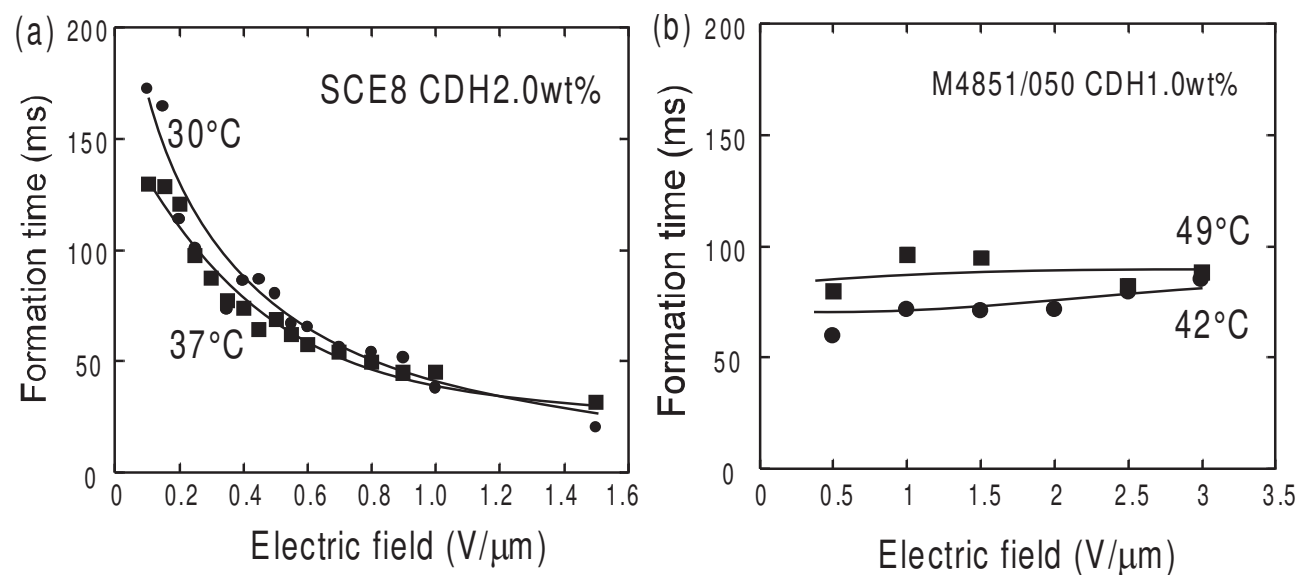

Figure 13. Electric field dependence of the index grating formation time. (a) SCE8 mixed with 2 wt $\%$ CDH and 0.1 wt

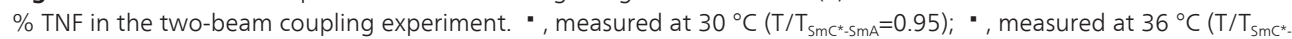
$\left.{ }_{\mathrm{smA}}=0.97\right)$. (b) M4851/050 mixed with $1 \mathrm{wt} \% \mathrm{CDH}$ and $0.1 \mathrm{wt} \% \mathrm{TNF}$ in a two-beam coupling experiment. " , measured at $42{ }^{\circ} \mathrm{C}\left(\mathrm{T} / \mathrm{T}_{\mathrm{SmC} \mathrm{C}^{*} \mathrm{SmA}}=0.95\right) ; \cdot$, measured at $49{ }^{\circ} \mathrm{C}\left(\mathrm{T} / \mathrm{T}_{\mathrm{SmC} \mathrm{C}^{*}-\mathrm{SmA}}=0.97\right)$.

\subsection{Formation mechanism of the internal electric field in FLCs}

Since the photorefractive effect is induced by the photoinduced internal electric field, the mechanism of the formation of the space-charge field in the FLC medium is important. The two-beam coupling gain coefficients of mixtures of FLC (SCE8) and photoconductive compounds under a DC field were investigated as a function of the concentration of TNF (electron acceptor). The photoconductive compounds - CDH, ECz and TNF (Figure 8) - were used in this examination. When an electron donor with a large molecular size (CDH) relative to the TNF was used as the photoconductive compound, the gain coefficient was strongly affected by the concentration of TNF (Figure 14(a)). However, when ethylcarbazole (ECz) - the molecular size of which is almost the same as that of TNF - was used, the gain coefficient was less affected by the TNF concentration (Figure 14(b)). 

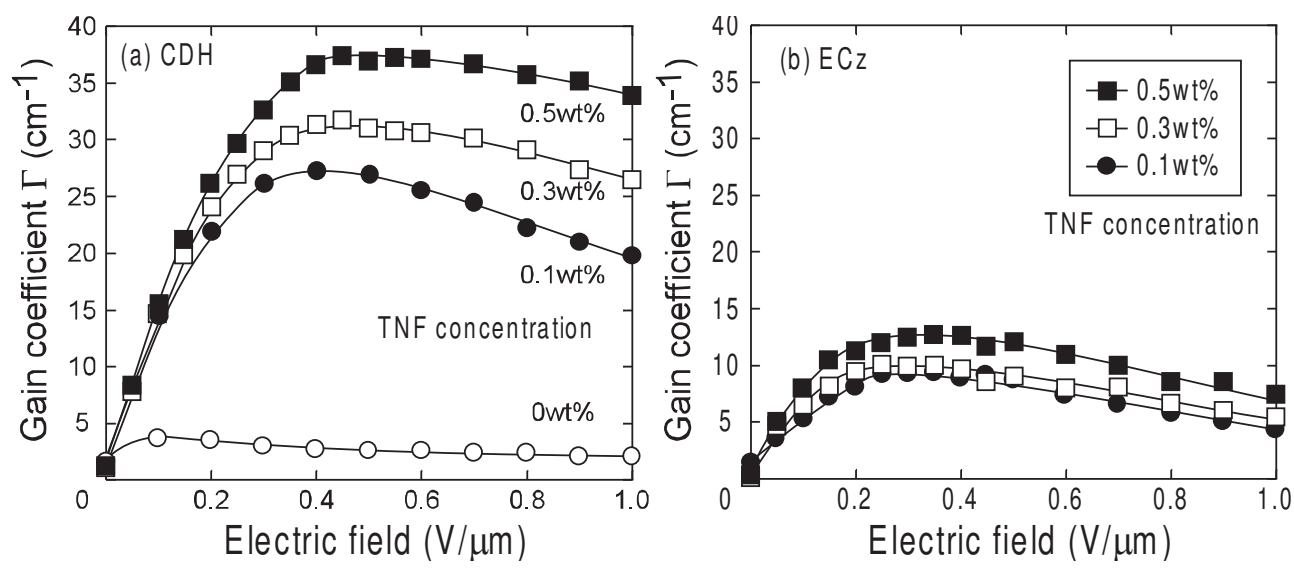

Figure 14. Dependence of the TNF concentration on the gain coefficients of an FLC doped with photoconductive dopants. (a) SCE8 doped with $2 \mathrm{wt} \% \mathrm{CDH}$, and (b) SCE8 doped with $2 \mathrm{wt} \% \mathrm{ECz}$. An electric field of $\pm 0.5 \mathrm{~V} / \mu \mathrm{m}, 100 \mathrm{~Hz}$ was applied.

These findings suggest that ionic conduction plays a major role in the formation of the space-charge field. The mobility of the $\mathrm{CDH}$ cation is smaller than that of the TNF anion, and this difference in mobility is thought to be the origin of the charge separation. In this case, the magnitude of the internal electric field is dominated by the concentration of the ionic species. On the other hand, the difference in the mobilities of ECZ and TNF is small and, thus, less effective charge separation is induced, indicating that the internal electric field is independent of the concentration of ionic species.

\subsection{Photorefractive effect in FLC mixtures containing photoconductive chiral compounds}

\subsubsection{Photoconductive chiral dopants}

The photorefractive effect of FLC mixtures containing photoconductive chiral dopants has been investigated [15]. The structures of the LC compounds, the electron acceptor trinitrofluorenone (TNF) and the photoconductive chiral compounds are shown in Figure 15. The mixing ratio of $8 \mathrm{PP} 8$ and $8 \mathrm{PP} 10$ was set to $1: 1$ because the $1: 1$ mixture exhibits the SmC phase over the widest temperature range. Hereafter, the 1:1 mixture of 8PP8 and 8PP10 is referred to as the base LC. The concentration of TNF was $0.1 \mathrm{wt} \%$. Four photoconductive chiral compounds with the terthiophene chromophore (3T-2MB, 3T-2OC, 3T-OXO, and 3TCF3) were synthesized. The base LC, TNF and a photoconductive chiral compound were dissolved in dichloroethane and the solvent was evaporated. The mixture was then dried in a vacuum at room temperature for one week. The samples were subsequently injected into a 10- $\mu$ m-gap glass cell equipped with $1-\mathrm{cm}^{2}$ ITO electrodes and a polyimide alignment layer for the measurements. The base LC, which is a 1:1 mixture of 8PP8 and 8PP10, was mixed with the photoconductive chiral dopants and the electron acceptor (TNF). The concentration of TNF was set to $0.1 \mathrm{wt} \%$. The terthiophene chiral dopants showed high 
miscibility with the phenyl pyrimidine-type smectic LC. The chiral smectic C $\left(\mathrm{SmC}^{*}\right)$ phase appeared in all of the mixtures of the base LC and the chiral dopants. With the increase of the concentration of the chiral dopants, the temperature range of the $\mathrm{SmC}^{*}$ phase and the chiral nematic $\left(\mathrm{N}^{*}\right)$ phase were reduced, whereas that of the smectic A (SmA) phase was enhanced. The miscibility of the 3T-CF3 with the base LC was the lowest among the four chiral dopants. It was considered that the dipole moment of the trifluoromethyl substituted group is large, so that the 3T-CF3 molecules tend to aggregate. All the samples exhibited absorption maxima at $394 \mathrm{~nm}$. Absorption at $488 \mathrm{~nm}$ (wavelength of the laser used) was small. The absorption spectra were not changed when TNF $(1.0 \times 10-5 \mathrm{~mol} / \mathrm{L})$ was added to the solution. The small absorption at the laser wavelength is advantageous for minimizing the optical loss. The photocurrents in the mixtures of the base LC, photoconductive chiral dopants and TNF were measured. As shown in Figure 16, the samples were good insulators in the dark. When a $488 \mathrm{~nm}$ laser irradiated the samples, photocurrents were clearly observed. The magnitudes of the photocurrents were slightly different in the four samples. The only difference in the molecular structures of these compounds is the chiral substituent. Thus, the difference in the photocurrent cannot be attributed to the difference in the molecular structure. It was considered that the miscibility of the photoconductive chiral compounds to the LC and the homogeneity of the LC phase affected the magnitude of the photocurrent.
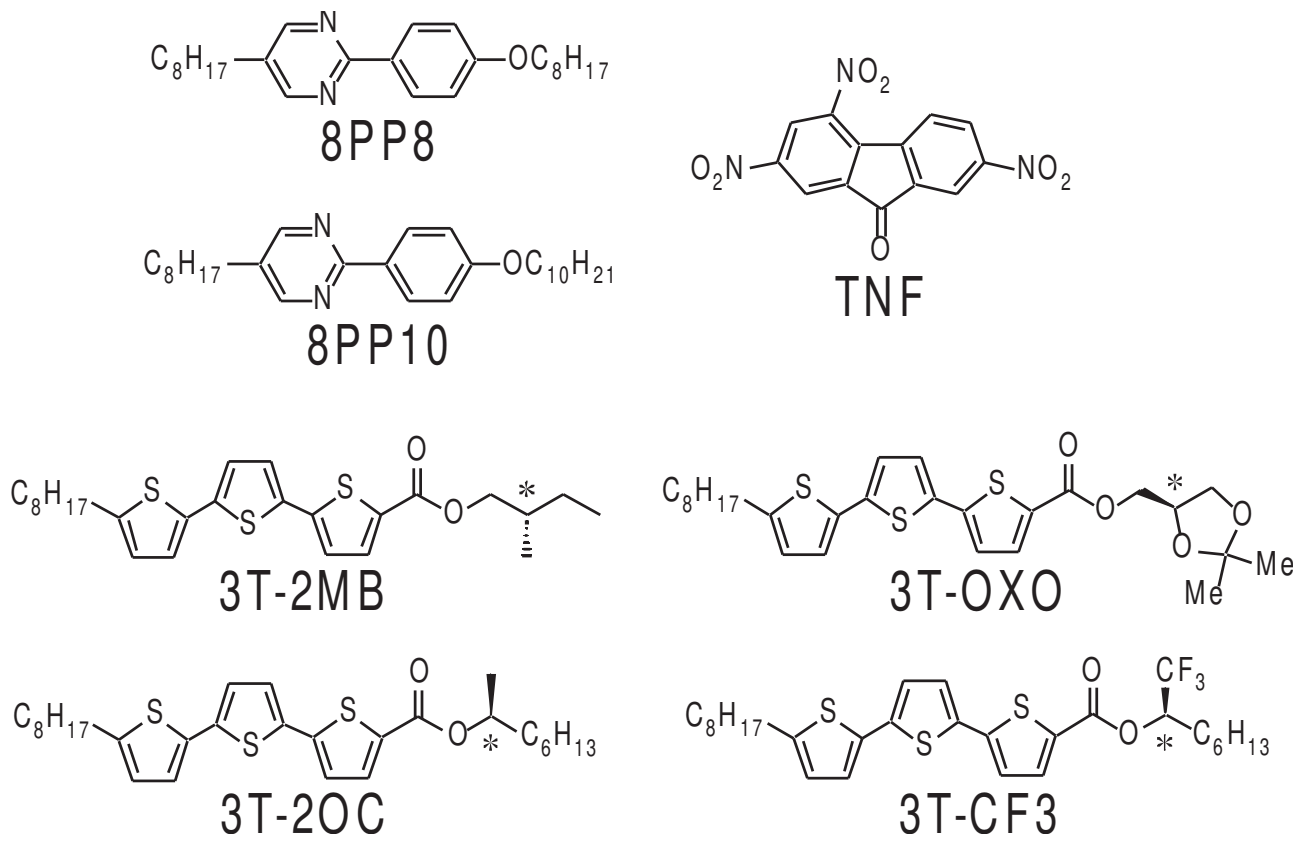

Figure 15. Structures of the smectic LCs (8PP8 and 8PP10), photoconductive chiral dopants (3T-2MB, 3T-2OC, 3T-OXO and 3 T-CF3) and the sensitizer TNF used in this work. 

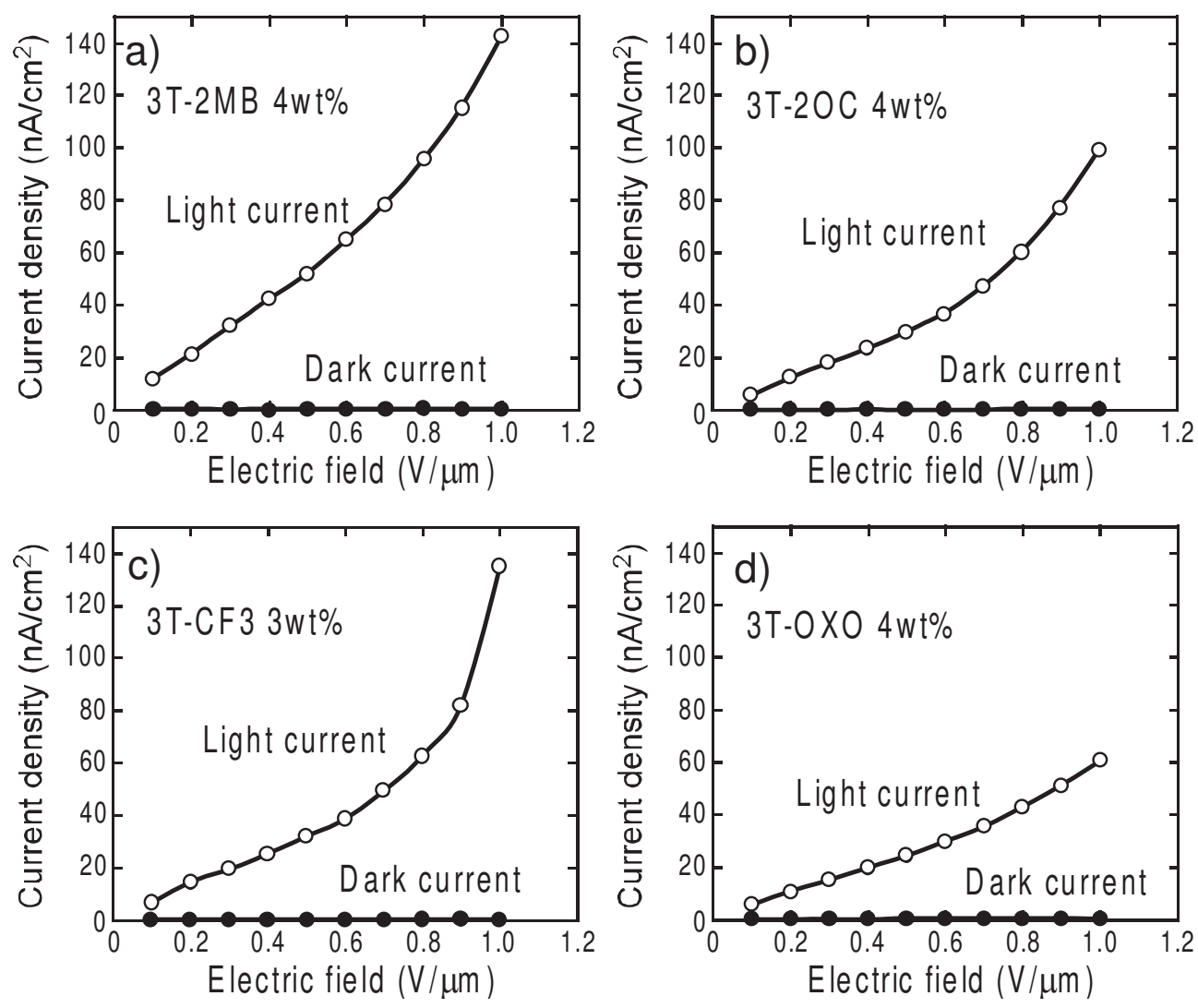

Figure 16. Magnitudes of light-current and dark-current of mixtures of the base LC, photoconductive chiral compound and TNF measured in a 10- $\mu \mathrm{m}$-gap LC cell as a function of the external electric field. An electric field of 0.1 $\mathrm{V} / \mu \mathrm{m}$ was applied. A $488 \mathrm{~nm} \mathrm{Ar}^{+}$laser $\left(10 \mathrm{~mW} / \mathrm{cm}^{2}, 1 \mathrm{~mm}\right.$ diameter $)$ was used as the irradiation source.

\subsubsection{Two-beam coupling experiment on photoconductive FLC mixtures}

The photorefractive effect was measured in a two-beam coupling experiment. A linearly polarized beam from an $\mathrm{Ar}^{+}$laser (488 nm, continuous wave) was divided into two by a beam splitter, each of which was then interfered in the sample film. A p-polarized beam was used. The laser intensity was $2.5 \mathrm{~mW}$ for each beam ( $1 \mathrm{~mm}$ diameter). The incident beam angles to the glass plane were $30^{\circ}$ and $50^{\circ}$. Each interval of the interference fringe was $1.87 \mu \mathrm{m}$. Figure 17 shows typical examples of the asymmetric energy exchange observed in a mixture of the base LC, 3T-2MB and TNF at $30^{\circ} \mathrm{C}$ with the application of an electric field of $0.2-0.4 \mathrm{~V} / \mu \mathrm{m}$. 


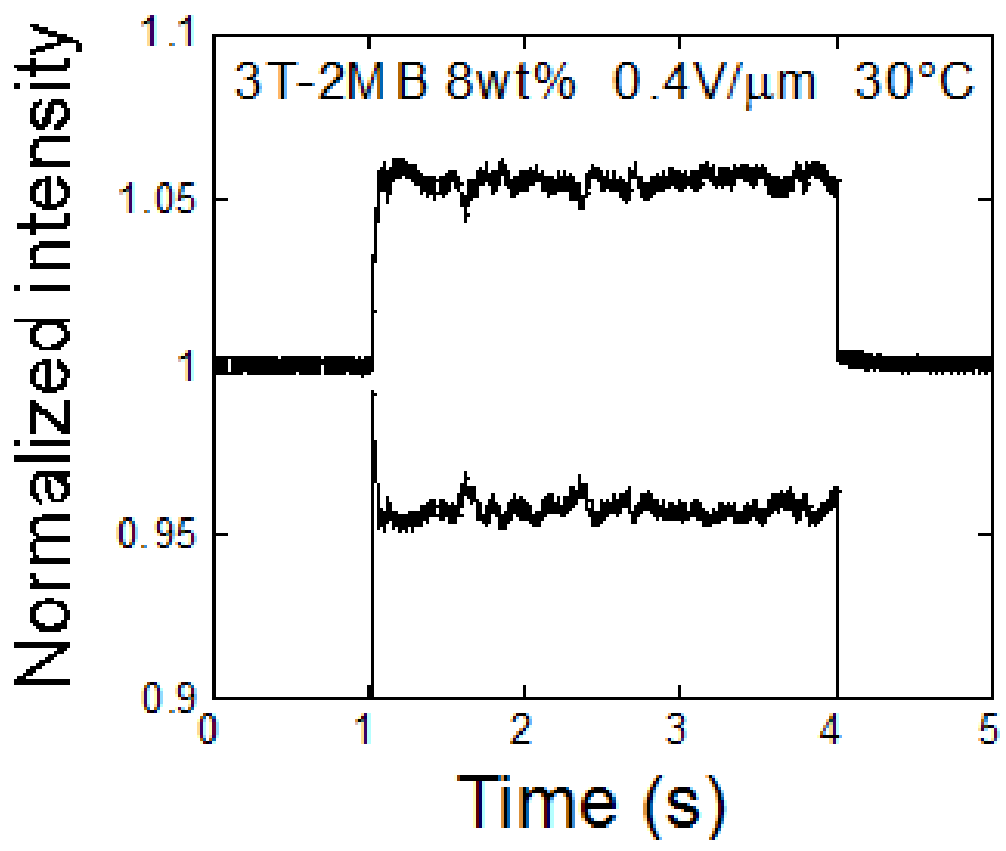

Figure 17. Examples of the results of two-beam coupling experiments for mixtures of the base LC, 3T-2MB and TNF measured at $30^{\circ} \mathrm{C}$.

The interference of the divided beams in the sample resulted in the increased transmittance of one of the beams and the decreased transmittance of the other beam. These transmittance characteristics were reversed when the polarity of the applied electric field was reversed. Asymmetric energy exchange was only observed when an electric field was applied, indicating that beam coupling was not caused by a thermal grating. With an increased concentration of $3 \mathrm{~T}-2 \mathrm{MB}$, the magnitude of the gain coefficient also increased. In order to calculate the twobeam coupling gain coefficient, the diffraction condition needs to be correctly identified. 

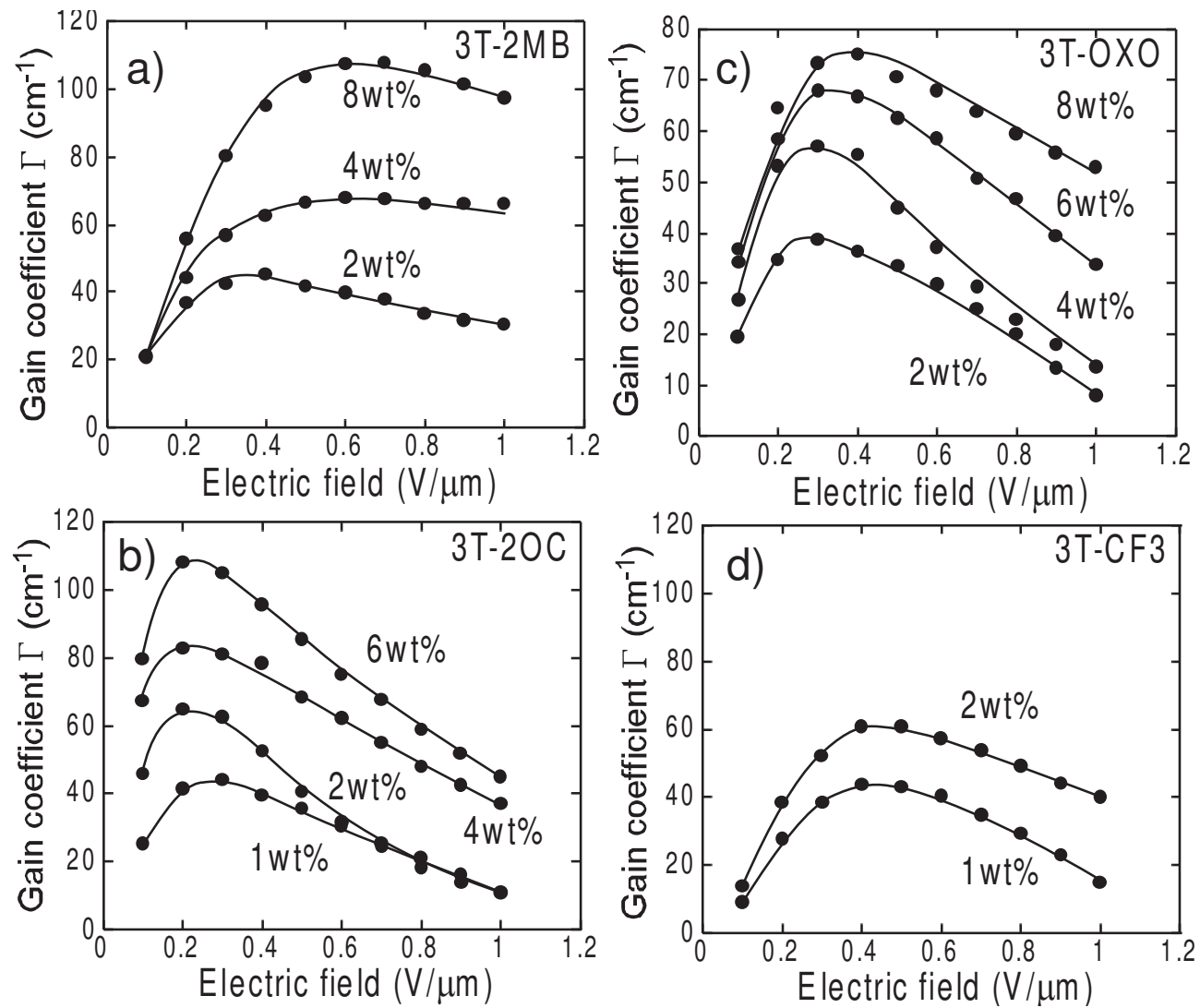

Figure 18. Electric field dependence of the gain coefficients of the mixtures of the base LC, photoconductive chiral compounds and TNF. (a) 3T-2MB; (b) 3T-2OC; (c) 3T-OXO; (d) 3T-CF3.

The difference in the gain coefficients in mixtures of the base LC, photoconductive chiral dopants (3T-2MB, 3T-2OC, 3T-OXO, and 3T-CF3) and TNF was investigated. All the samples formed a finely aligned SS-state in 10- $\mu$ m-gap cells with a LX-1400 polyimide alignment layer and exhibited clear photorefractivity in the ferroelectric phase. The asymmetric energy exchange was only observed in the temperature range in which the sample exhibits ferroelectric properties ( $\mathrm{SmC}^{*}$ phase). The gain coefficients of the samples are plotted as a function of the magnitude of the external electric field in Figure 18. The gain coefficient increased with the strength of the external electric field up to 0.2-0.6 V/ $\mu \mathrm{m}$ and then decreased with the strength of the external electric field. As the concentration of the photoconductive chiral dopants increased, so did the gain coefficient. This may be due to the increased density of charge carriers in the FLC medium and an increase in the magnitude of Ps. All the samples exhibited relatively large photorefractivity. A gain coefficient higher than $100 \mathrm{~cm}^{-1}$ was obtained in the 3T-2OC (6 $\mathrm{wt} \%)$ sample with the application of only $0.2 \mathrm{~V} / \mu \mathrm{m}$. This means that a voltage of only $2 \mathrm{~V}$ is needed to obtain the gain coefficient of $100 \mathrm{~cm}^{-1}$ in a $10 \mu \mathrm{m}$ FLC sam- 
ple. In the FLCs reported previously, gain coefficients of only $50-60 \mathrm{~cm}^{-1}$ were obtained with the application of a $1 \mathrm{~V} / \mu \mathrm{m}$ electric field. A gain coefficient higher than $100 \mathrm{~cm}^{-1}$ was also obtained in the 3T-2MB sample with an applied electric field of $0.5 \mathrm{~V} / \mu \mathrm{m}$. In order to obtain photorefractivity in polymer materials, the application of a high electric field of $10-50 \mathrm{~V} / \mu \mathrm{m}$ to the polymer film is typically required. The small electric field necessary for the activation of the photorefractive effect in FLCs is thus a great advantage for their use in photorefractive devices. The miscibility of 3T-CF3 with the base LC was low and could be mixed with the base LC at concentrations lower than $2 \mathrm{wt} \%$. The grating formation time in the mixtures of the base LC, photoconductive chiral dopants and TNF is plotted as a function of the strength of the external electric field in Figure 19. The grating formation time decreased with an increased electric field strength due to the increased efficiency of charge generation. The shortest formation time was obtained as $5-8 \mathrm{~ms}$ with a $1 \mathrm{~V} / \mu \mathrm{m}$ external electric field in all chiral compounds. The 3T-CF3 sample exhibited the fastest response. This was because of the larger polarity of the 3T-CF3.
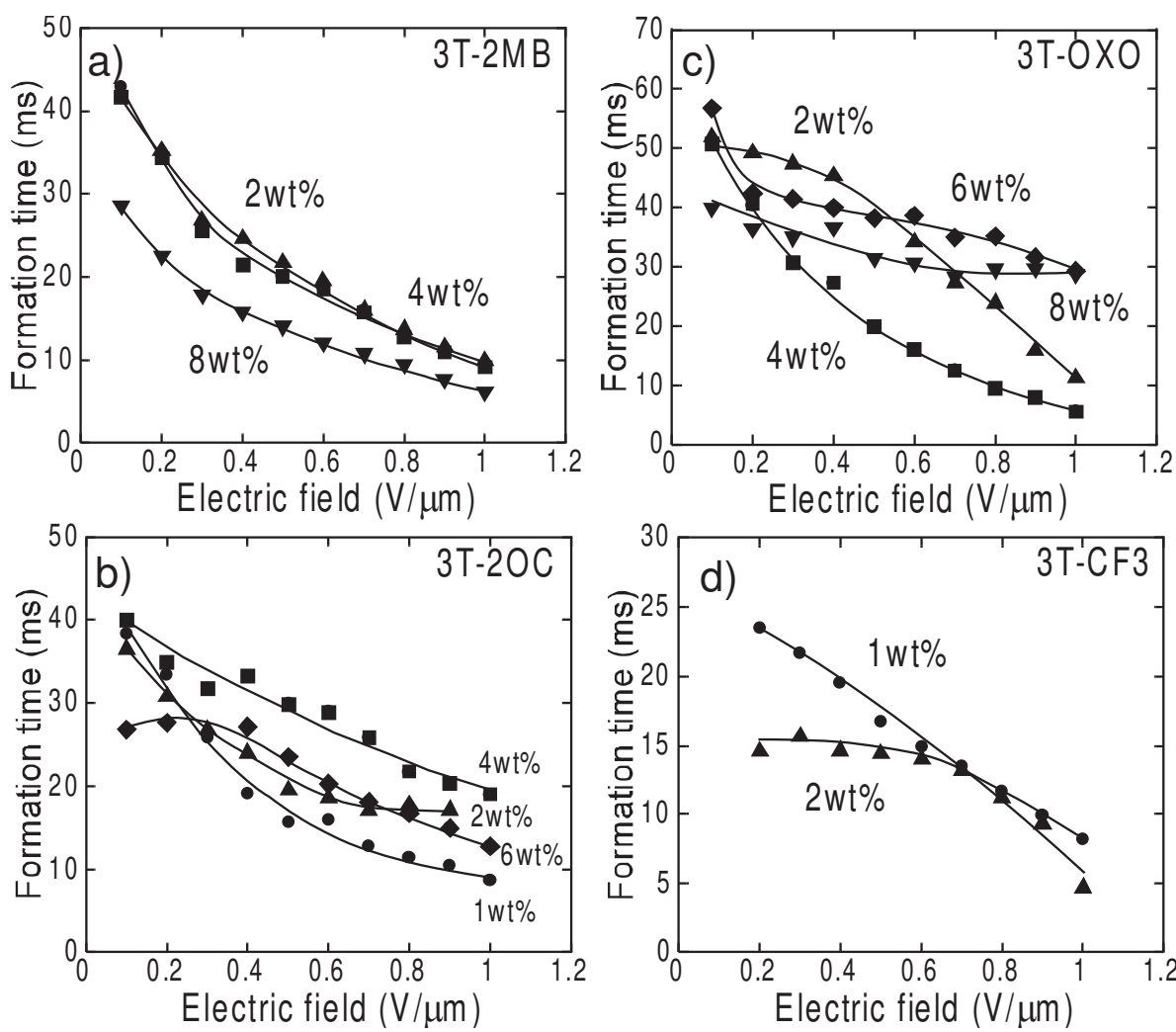

Figure 19. Electric field dependence of the index grating formation times of mixtures of the base LC, photoconductive chiral compounds and TNF measured at $30^{\circ} \mathrm{C}$. (a) 3T-2MB; (b) 3T-2OC; (c) 3T-OXO; (d) 3T-CF3. 


\subsubsection{Photorefractive effect in a ternary mixture of a SmC liquid crystal doped with the photoconductive chiral compound}

The photorefractive effect of the mixture shown in Figure 20 was investigated. The gain coefficients of the samples were measured as a function of the applied electric field strength (Figure 21(a)). The gain coefficient was calculated to be higher than $800 \mathrm{~cm}^{-1}$ in the $10 \mathrm{wt} \%$ sample with the application of only $1 \mathrm{~V} / \mu \mathrm{m}$. This gain coefficient is eight times higher than that of the FLCs described in the previous section. It was considered that a higher transparency of the ternary LC mixture contributed to the high gain coefficient. The small electric field required to activate the photorefractive effect in FLCs is a great advantage for photorefractive devices. The response time decreased with an increased electric field strength due to the increased charge separation efficiency (Figure 21(b)). The shortest formation time obtained was $8 \mathrm{~ms}$ for an external electric field of $1.5 \mathrm{~V} / \mu \mathrm{m}$. The large gain and fast response are advantageous for realizing optical devices, such as real-time image amplifiers and accurate measurement devices.

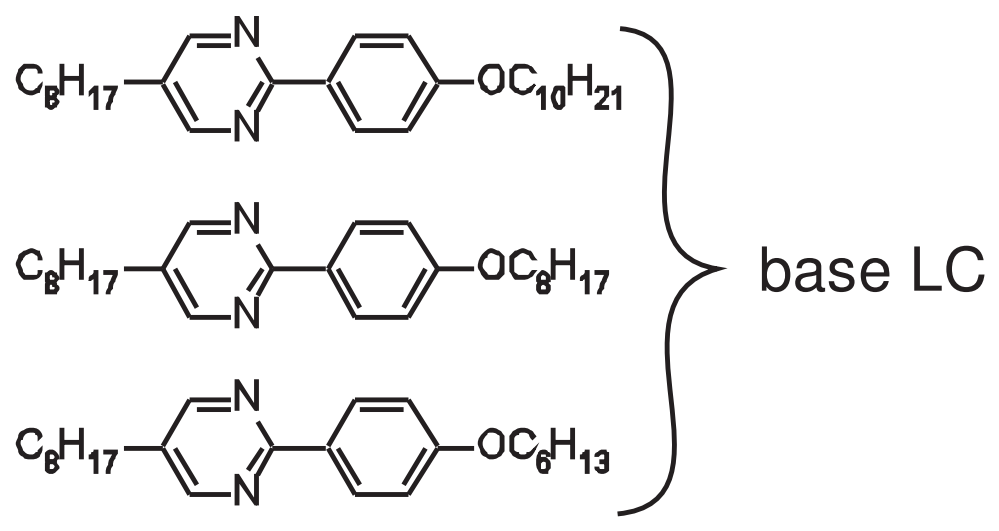<smiles>C[CH]c1ccc(-c2ccc(-c3ccc(C(=O)OCC=C(C)CC)o3)s2)s1</smiles><smiles>O=C1c2cc([N+](=O)[O-])ccc2-c2c1cc([N+](=O)[O-])cc2[N+](=O)[O-]</smiles>

Figure 20. Photorefractive FLC mixture containing a ternary mixture of smectic LCS. 
(a)

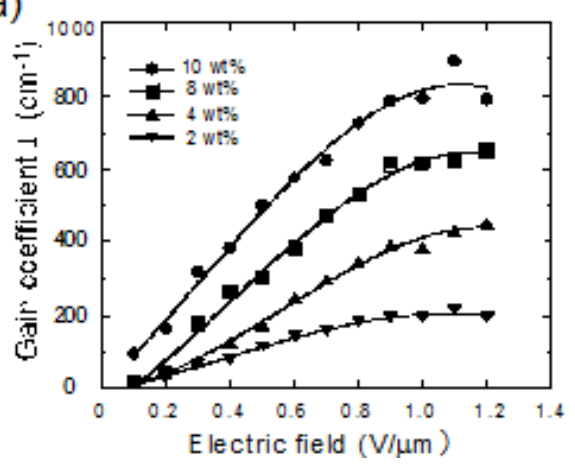

(b)

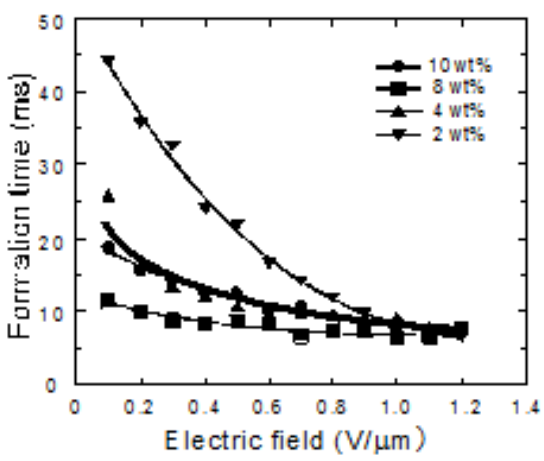

Figure 21. (a) Electric field dependence of gain coefficients of mixtures of the base LC, 3T-2MB and TNF (0.1 wt\%) measured at $30^{\circ} \mathrm{C}$. The $3 \mathrm{~T}-2 \mathrm{MB}$ concentration was within the range $2-10 \mathrm{wt} \%$. (b) Refractive index grating formation time (response time) of mixtures of the base LC, $3 \mathrm{~T}-2 \mathrm{MB}$ and TNF $(0.1 \mathrm{wt} \%)$ measured at $30^{\circ} \mathrm{C}$. The $3 \mathrm{~T}-2 \mathrm{MB}$ concentration was within the range $2-10 \mathrm{wt} \%$.

\subsubsection{Formation of dynamic holograms in FLC mixtures}

The formation of a dynamic hologram was demonstrated. A computer-generated animation was displayed on a spatial light modulator (SLM). A $488 \mathrm{~nm}$ beam from a DPSS laser was irradiated on the SLM and the reflected beam was incident on the FLC sample. A reference beam interfered with the beam from the SLM in the FLC sample. The refractive index grating formed was within the Raman-Nath regime, in which multiple scattering is allowed. A $633 \mathrm{~nm}$ beam from a He-Ne laser was irradiated on the FLC sample and the diffraction was observed. A moving image of the animation on the computer monitor was observed in the diffracted beam (Figure 22). No image retention was observed, which means that the hologram image (refractive index grating) formed in the FLC was rewritten sufficiently rapidly to project the reproduction of a smooth holographic movie. 

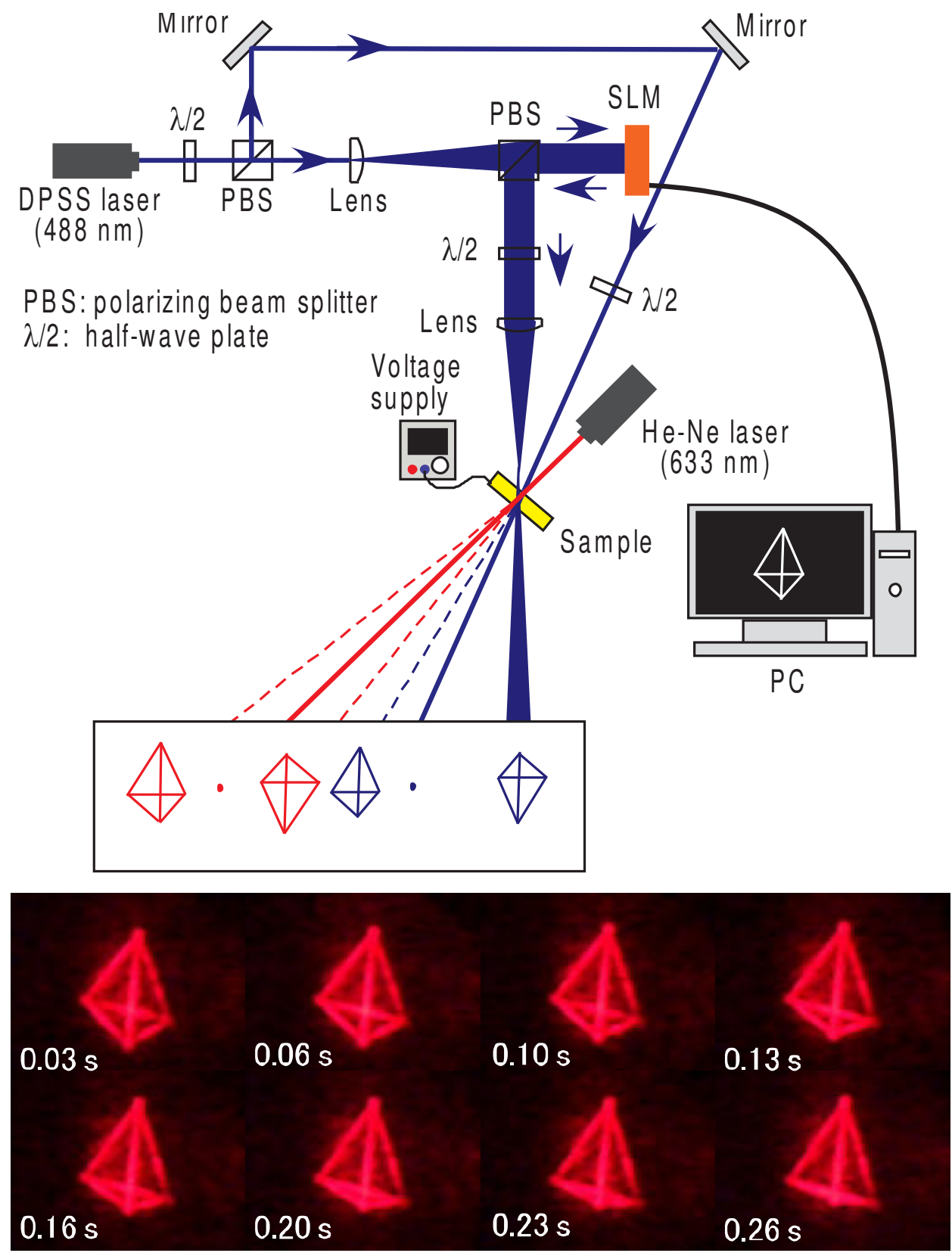

Figure 22. Dynamic hologram formation experiment on an FLC sample. A computer-generated animation was displayed on the SLM. The SLM modulated the object beam $(488 \mathrm{~nm})$, which was irradiated on the FLC sample and interfered with the reference beam. The readout beam $(633 \mathrm{~nm})$ was irradiated on the FLC and diffraction was observed. 


\section{Photorefractive effect in FLCs with the application of an AC field}

\subsection{Formation of dynamic holograms based on the spatial modulation of the molecular motion of FLCs}

The formation of dynamic holograms based on the spatial modulation of the molecular motion of ferroelectric liquid crystals (FLCs) was demonstrated [16, 17]. The switching movement of an FLC molecule is essentially a rotational motion along a conical surface. When an alternating triangular-waveform voltage is applied, the FLC molecules uniformly go through a consecutive rotational switching motion along a conical surface (Figure 23(a)). If a photoconductive FLC material is used, this rotational motion can be modulated by illuminating the material with interfering laser beams, as shown in Figure 23. The internal electric field vector is directed along the interference fringe wave vector and, in many cases, it differs from the direction of the applied alternating electric field. Thus, the total electric field at each moment on the FLC molecules is altered by the presence of the internal electric field. The consecutive rotational motion of the FLC molecules in the areas between the light and dark positions of the interference fringes is biased by the internal electric field. Consequently, a grating based on the spatial difference in the rotational motion (or switching motion) of the FLC molecules is created. This grating is different from those currently used for holograms, wherein changes in the static properties of a medium - such as absorbance, transparency, film thickness and molecular orientation - are induced by photochemical reactions.

The formation of a motion-mode grating was examined using a SCE8/CDH/TNF mixture in a $10 \mu \mathrm{m}$-gap-cell. The formation of a holographic grating and the occurrence of a phase shift between the formed grating and the interference fringes were examined in this experiment. An alternating triangular-waveform electric field ( 0 to $\pm 1 \mathrm{~V} / \mu \mathrm{m}$, and $1 \mathrm{kHz}$ to $3 \mathrm{MHz}$ ) was applied to the sample. Under the effect of an alternating triangular-waveform electric field of $\pm 0.5 \mathrm{~V} / \mu \mathrm{m}$ $100 \mathrm{kHz}$ in the two-beam coupling experiment - the FLC molecules exhibited a consecutive switching motion. This switching was confirmed using a polarizing microscope equipped with a photodetector Figure 24. Figure 25(a) shows the transmitted intensities of the laser beams through the FLC/CDH/TNF mixture upon the application of an alternating electric field as a function of time. The interference of the divided beams in the sample resulted in the increased transmittance of one of the beams and the decreased transmittance of the other. The incident beam conditions were the same as those used in the DC experiment.

Although the transmitted intensity of the laser beam oscillates due to the switching motion of the FLC molecules, the average intensities of the beams were symmetrically changed, as shown in Figure 25. This indicates that a diffraction grating based on the spatial difference in the rotational switching motion of FLC molecules was formed. The symmetric change in the transmittance of the two beams proves that the phase of the motion-mode grating shifted from that of the interference fringe. No higher-order diffraction was observed under this condition. The spacing of the grating was calculated to be $0.9 \mu \mathrm{m}(1,100$ lines $/ \mathrm{mm})$. Figure 26 shows the temperature dependence of the gain coefficient obtained under an AC electric field. Asymmetric energy exchange was observed only at those temperatures at which the FLC/CDH/TNF mixture exhibits a ferroelectric phase. This suggests that ferroelectricity or the switching movement of $\mathrm{SmC}^{*}$ is necessary for beam coupling under an AC electric field. 
(a)

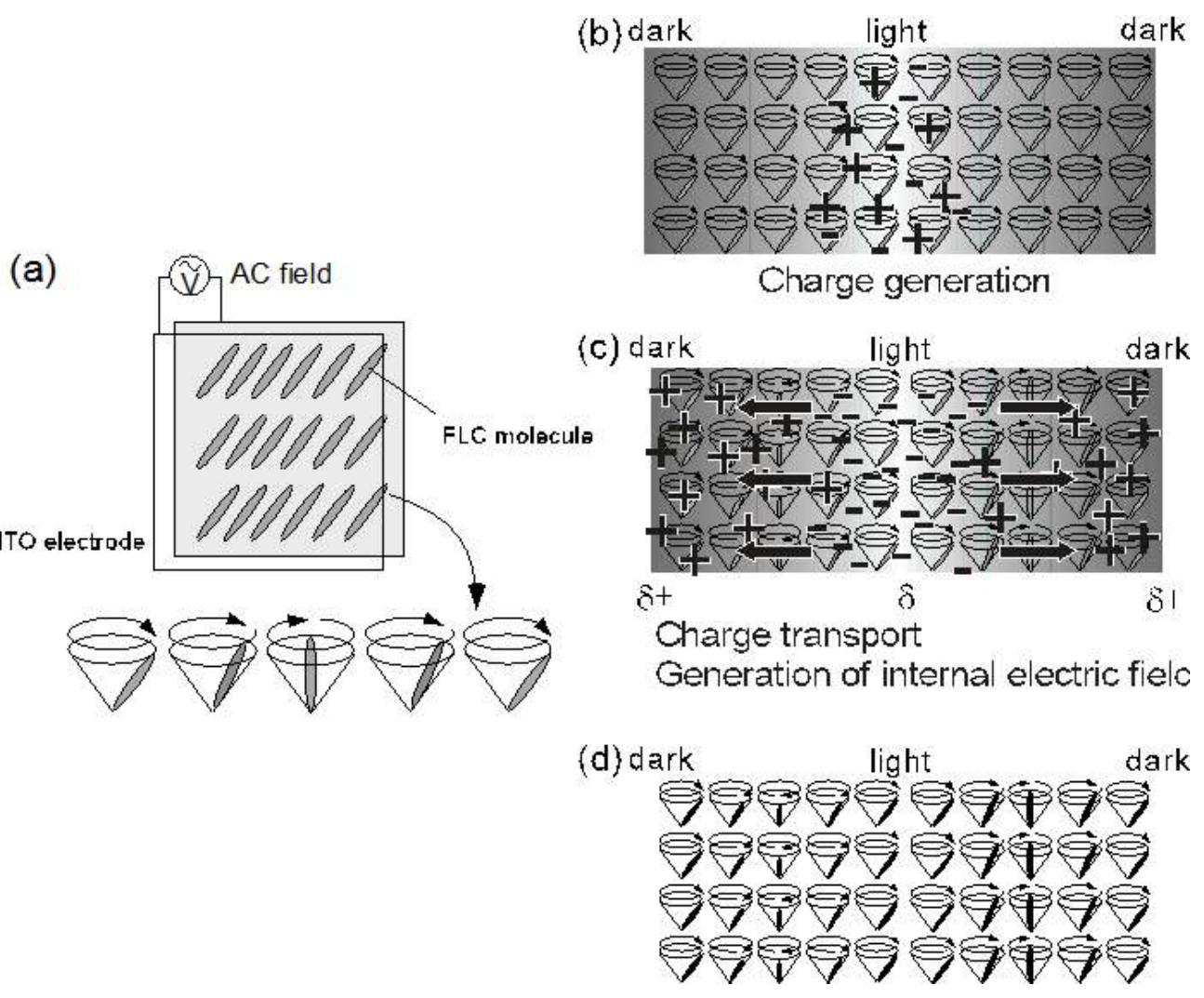

Figure 23. (a) Electro-optical switching of an FLC. (b) The rotational motion of FLC molecules under the application of an alternating electric field. (c) Positive and negative charges appear at the light positions of the interference fringe. (d) An internal electric field develops in the area between the light and dark positions of the interference fringes. The rotational motion of the FLC molecules in the corresponding area is biased by the internal electric field. 


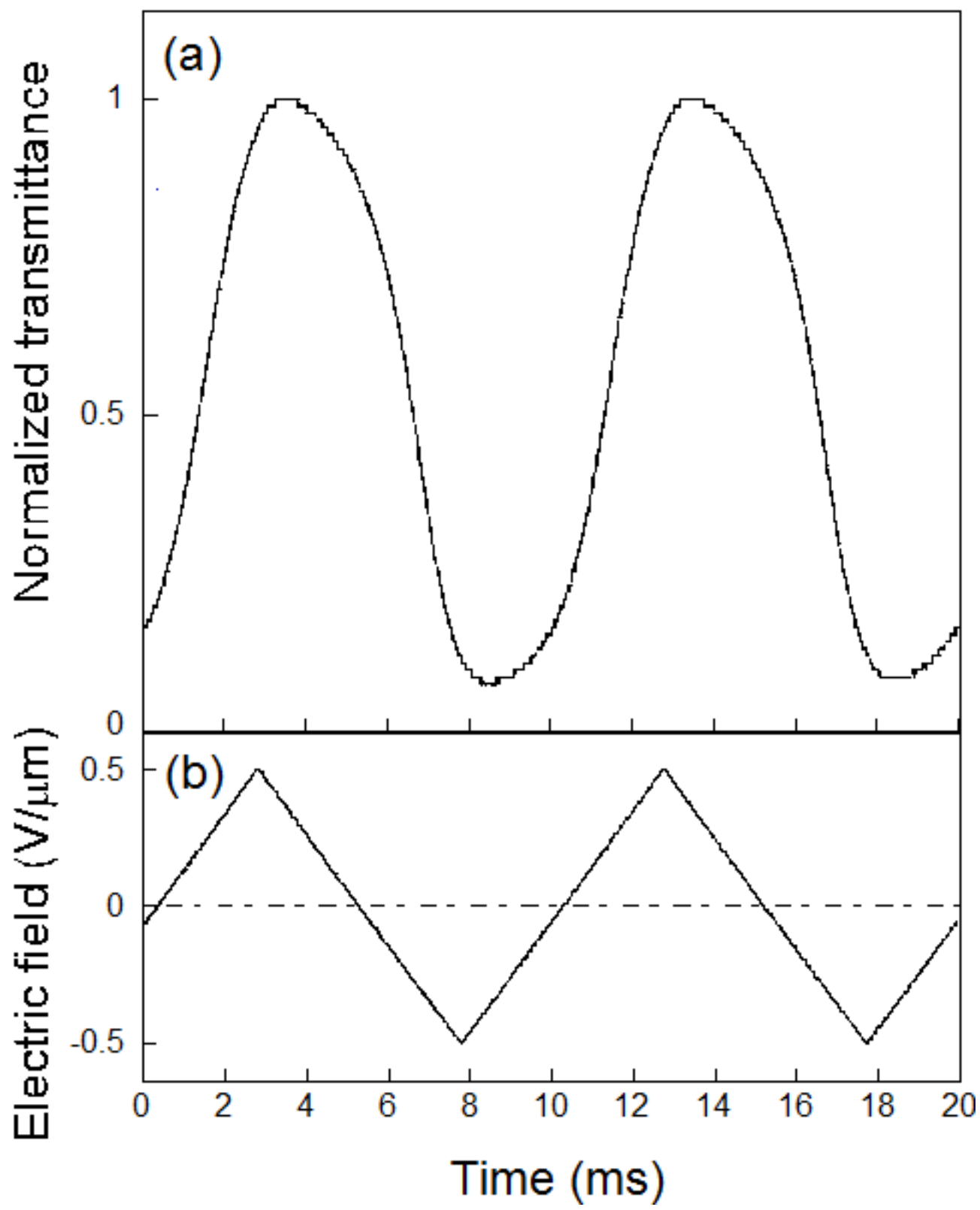

Figure 24. Switching behaviour of SCE8 mixed with $2 \mathrm{wt} \% \mathrm{CDH}$ and $0.1 \mathrm{wt} \% \mathrm{TNF}$ under a $100 \mathrm{~Hz}, \pm 0.5 \mathrm{Vm} / \mu \mathrm{m}$ triangular wave electric field. (a) Transmittance of light through the FLC under a polarizing microscope. (b) Magnitude of the applied electric field. 

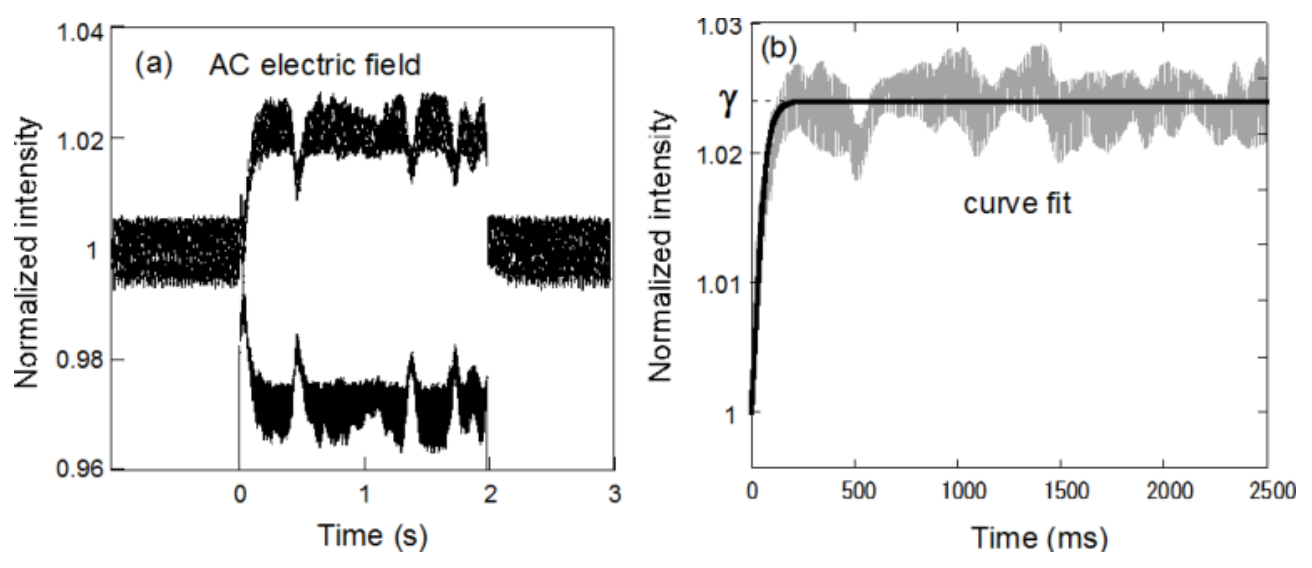

Figure 25. a) Typical example of the asymmetric energy exchange observed in two-beam coupling experiments. A triangular waveform AC electric field of $\pm 0.5 \mathrm{Vm} / \mu \mathrm{m}, 100 \mathrm{~Hz}$ was applied in this case. The sample angle a was $50^{\circ}$ and the intersection angle $\phi$ was $20^{\circ}$. The shutter was opened at $t=0 \mathrm{~s}$ and closed at $t=2 \mathrm{~s}$. (b) An example of the curve fit.

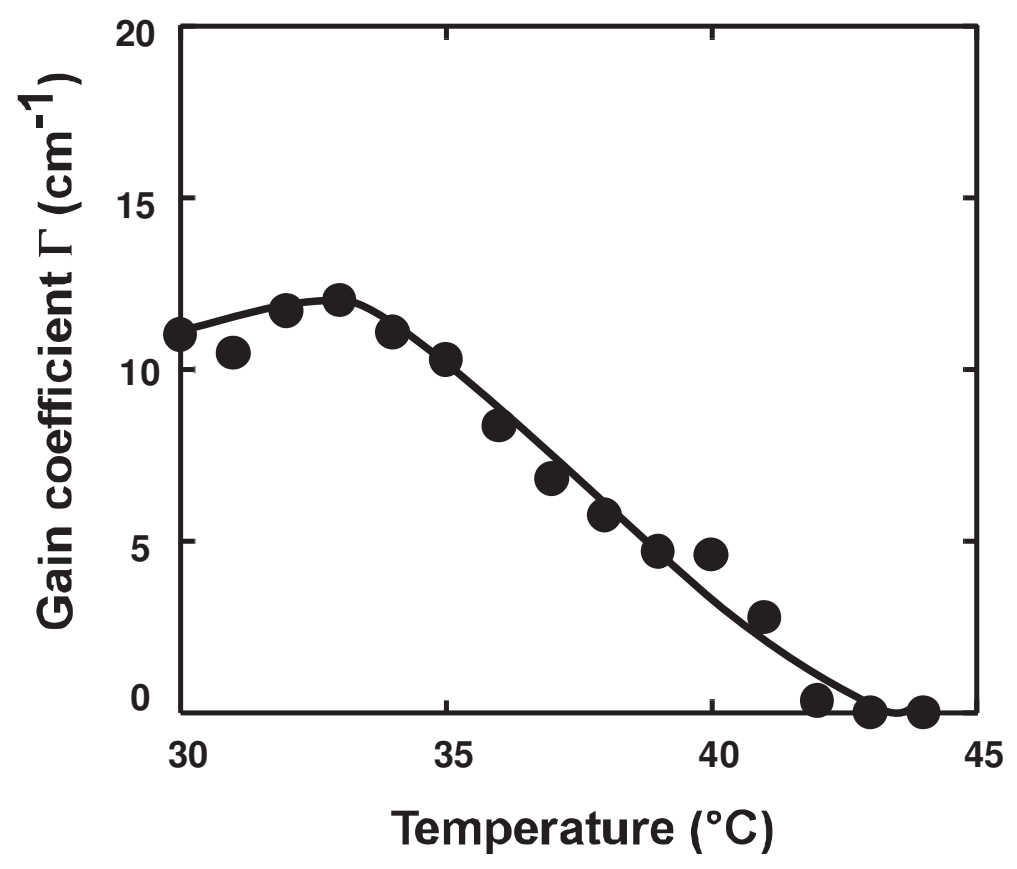

Figure 26. Gain coefficient of the two-beam coupling under an AC electric field as a function of temperature. An AC electric field of $\pm 0.5 \mathrm{~V} / \mu \mathrm{m}, 100 \mathrm{~Hz}$ was applied. 
Figure 27 shows the gain coefficient plotted as a function of the electric field strength. Asymmetric energy exchange was observed at electric field strengths higher than $\pm 0.1 \mathrm{~V} / \mu \mathrm{m}$. Furthermore, no asymmetric energy exchange was observed without an external electric field. This eliminates the possibility that beam coupling resulted from either a thermal grating or from a photochemically-formed grating. The gain coefficient was independent of the external electric field strength for fields higher than $\pm 0.1 \mathrm{~V} / \mu \mathrm{m}$. This behaviour differs from that reported previously for the photorefractive effect of FLCs under an applied DC electric field, wherein the gain coefficient decreased with the increasing strength of the external DC electric field. The refractive index grating formation time was measured based on the simplest single-carrier model for photorefractivity, whereby the gain transient is exponential. The grating formation time $\tau$ was obtained from the fitted curve.

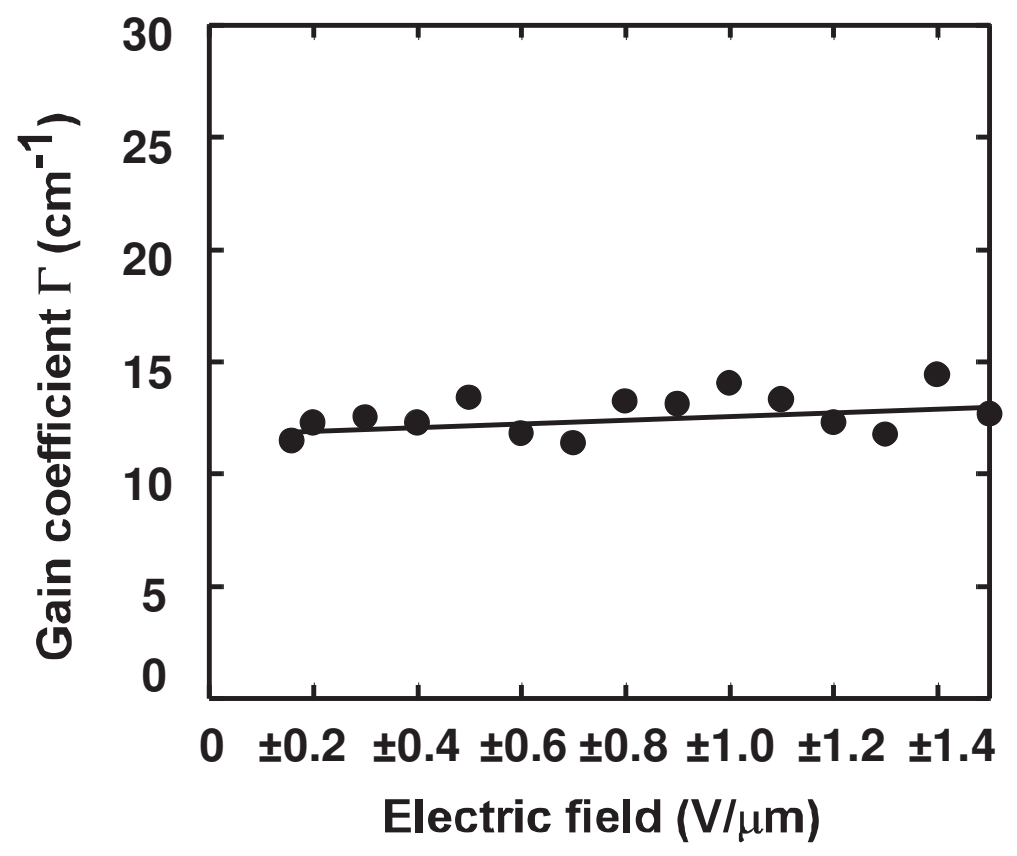

Figure 27. Gain coefficient of the two-beam coupling as a function of the applied AC electric field strength. The frequency of the field was $100 \mathrm{~Hz}$. 


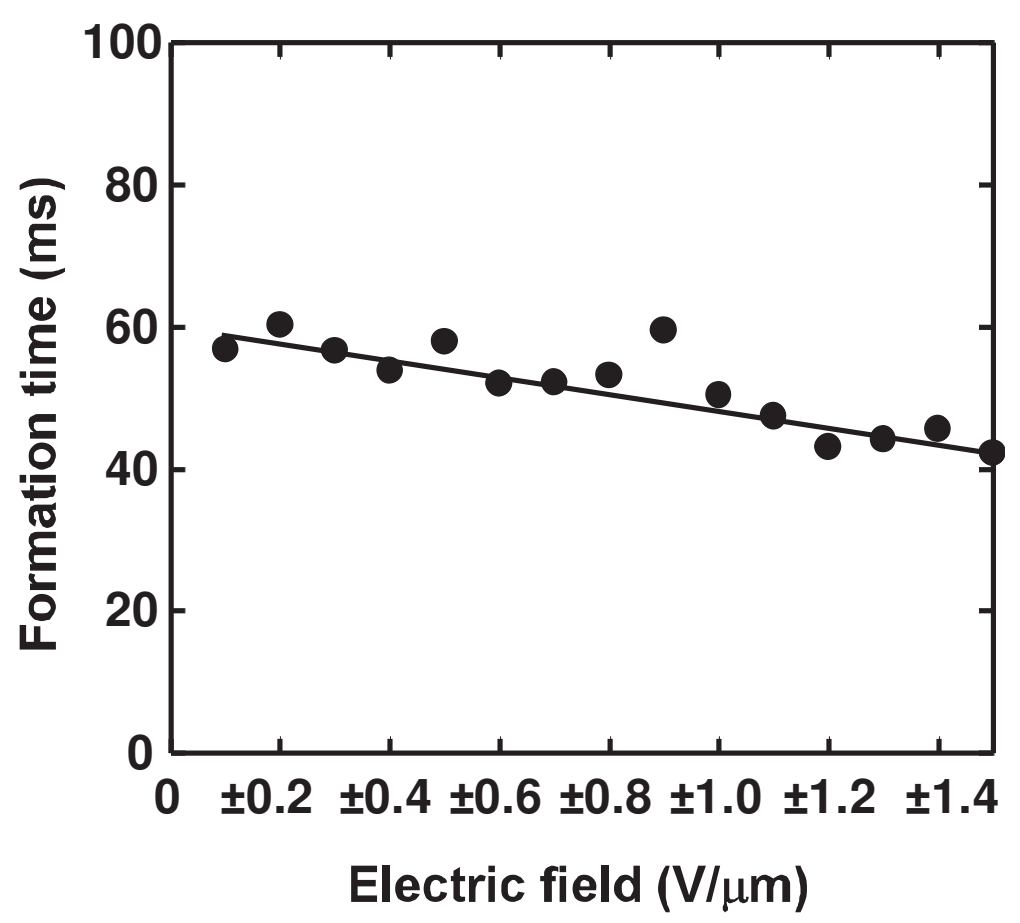

Figure 28. Grating formation time as a function of the AC electric field strength. The frequency of the AC field was $100 \mathrm{~Hz}$.

As seen in Figure 28, the grating formation time decreased with an increased applied electric field strength as a result of the increased efficiency of charge generation. The grating formation time was determined to be $30-40 \mathrm{~ms}$ in the present case.

\subsection{Frequency dependence of the gain coefficient and the grating formation time}

The gain coefficients are plotted as a function of the frequency of the applied external electric field in Figure 29.

The gain coefficient was observed to increase with an increase in frequency within the range of $1 \mathrm{~Hz}$ to $100 \mathrm{~Hz}$. The FLC exhibited switching based on polarization reversal at a frequency lower than $500 \mathrm{~Hz}$. The gain coefficient reached a maximum at frequencies within the range of $60 \mathrm{~Hz}$ to $500 \mathrm{~Hz}$. However, the magnitude of the gain coefficient decreased as the frequency was increased to higher than $500 \mathrm{~Hz}$. This is thought to be because the FLC cannot perform a complete switching motion at frequencies higher than $500 \mathrm{~Hz}$; therefore, the FLC molecules showed vibrational motion. Moreover, at frequencies from $500 \mathrm{~Hz}$ to 2000 $\mathrm{Hz}$, some of the FLC molecules go through a rotational switching motion, whereas others undergo vibrational motion. When the frequency exceeded $2000 \mathrm{~Hz}$, the magnitude of the gain coefficient reaches a constant value, regardless of the frequency. Thus, the value of the 
gain coefficient at frequencies lower than $500 \mathrm{~Hz}$ can be attributed to the photorefractive effect based on the complete switching of FLC molecules and, at frequencies higher than 2000 $\mathrm{Hz}$, this represents the photorefractive effect based on the vibrational motion of FLC molecules caused by an alternating electric field. Figure 30 shows the frequency dependence of the grating formation time.

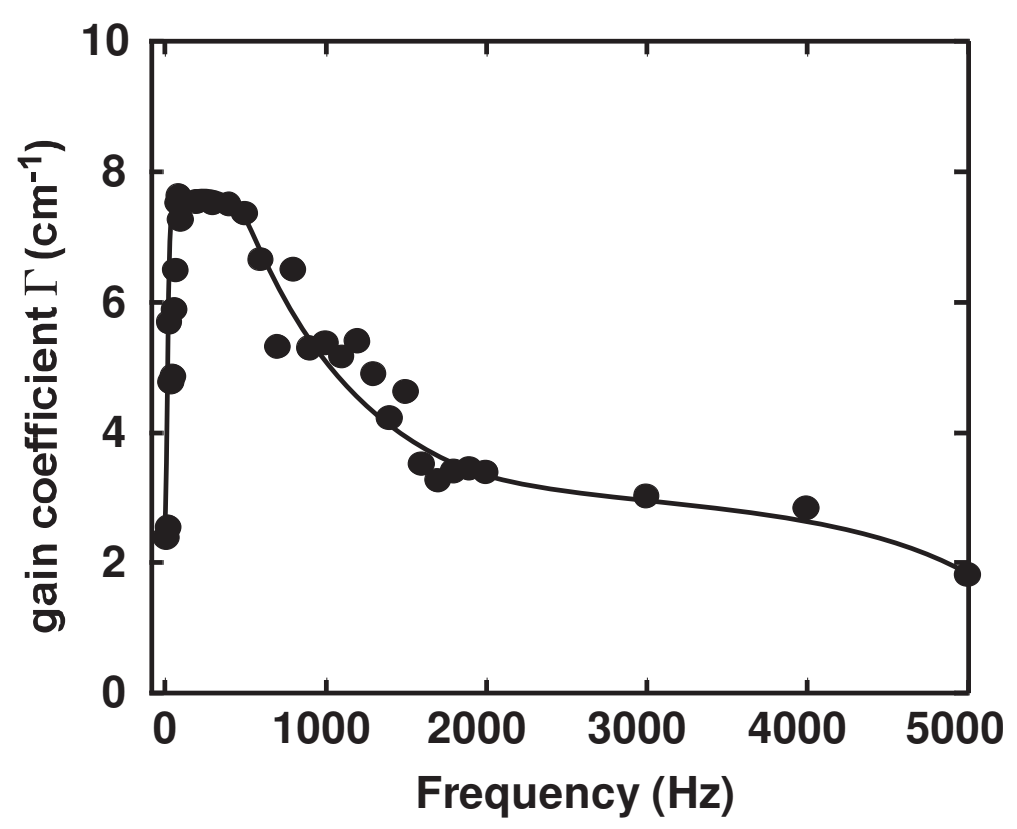

Figure 29. Gain coefficient as a function of the frequency of the applied electric field. The frequency was varied from 1 to $5000 \mathrm{~Hz}$. The strength of the field was $\pm 0.5 \mathrm{~V} / \mu \mathrm{m}$. 


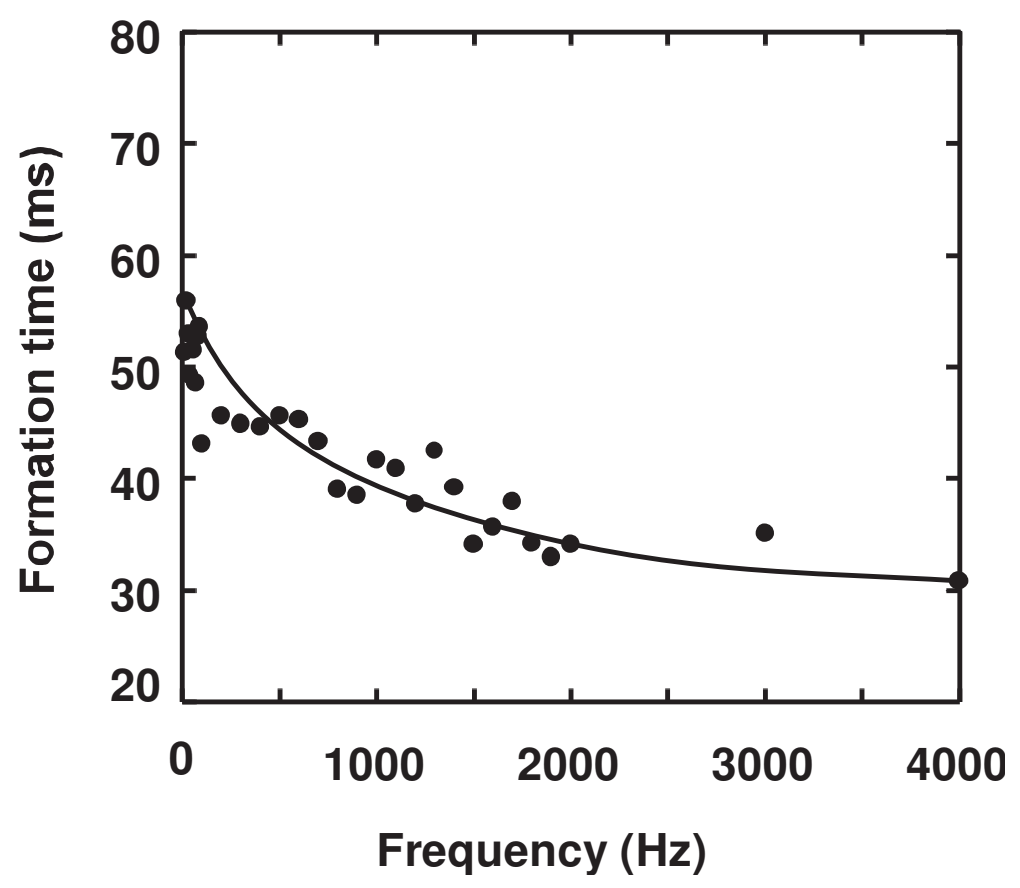

Figure 30. Grating formation time as a function of the frequency of the applied electric field. The frequency was varied from $1 \mathrm{~Hz}$ to $4000 \mathrm{~Hz}$. The strength of the field was $\pm 0.5 \mathrm{~V} / \mu \mathrm{m}$.

The grating formation time was shortened as the frequency increased. In addition, the frequency dependence of the formation time was not correlated with the dependence of the gain coefficient. As the frequency of the applied electric field increased, the switching and vibrational motion of the FLC molecules is thought to be accelerated and the affect on the internal electric field becomes apparent. Asymmetric energy exchange in FLCs under a static DC field is dominated by the direction of the DC field. The increase/decrease in the beam intensity switches when the direction of the field is reversed. This originates from the direction of charge separation. Thus, an internal electric field is thought to be difficult to form under an AC electric field. However, asymmetric energy exchange was observed with good reproducibility, suggesting that an internal electric field is formed even under an applied AC electric field. In addition, no energy exchange was observed in any FLC lacking a photoconductive dopant under an AC electric field (Figure 31). This observation indicates that photoconductivity is necessary for two-beam coupling under an AC field. 


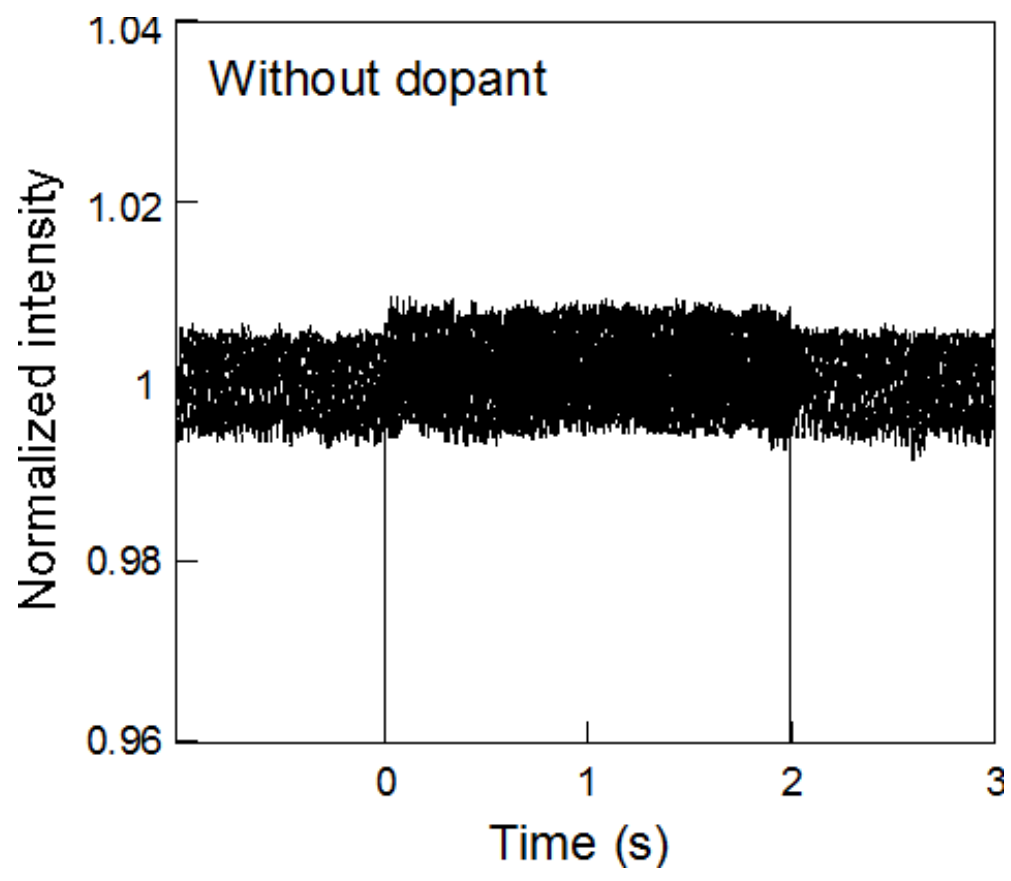

Figure 31. An example of the results of the two-beam coupling experiment under an AC electric field for SCE8 without a photoconductive dopant. An AC electric field of $\pm 0.5 \mathrm{~V} / \mu \mathrm{m}, 100 \mathrm{~Hz}$ was applied. The shutter was opened at $\mathrm{t}=$ $0 \mathrm{~s}$ and closed at $\mathrm{t}=2 \mathrm{~s}$.

It is necessary to consider both hole transport by a hopping mechanism as well as ionic conduction in order to explain the formation of the space-charge field under an AC field. In addition, a number of experimental results support the large contribution of ionic conduction to the FLC medium. In addition, the photorefractive effect in SCE8 doped with $2 \mathrm{wt} \% \mathrm{ECz}$ under an AC field was very small. If ionic conduction is the major contributor to the formation of the space-charge field, the anisotropic mobility of ionic species in the LC medium may affect the formation of the field. As shown in Figure 3, interference fringes are formed across the smectic layer so that migration of ionic species occurs in the inter-layer direction. The asymmetric structure of the surface stabilized state of the FLC may lead to the asymmetric mobility of cations and anions, which is thought to be one possible model explaining the charge separation under an AC electric field. However, the mechanism of the formation of the space-charge field under an AC field requires further investigation.

\subsection{Two beam coupling experiment with the application of a biased AC field}

An asymmetric energy exchange was observed upon the application of an AC field. This grating was interpreted as being based on the spatial difference in the molecular motion of the FLC molecules. The response time was on the order of a few tens of milliseconds and was dominated by the formation of the internal electric field. The photorefractivity in FLCs 
is accomplished through charge generation and diffusion. The application of an AC field to an FLC results in a very stable photorefractive response. However, it is obvious that an AC field is not advantageous for charge separation. Thus, the effect of a biased AC electric field on the photorefractivity of SS-FLCs was investigated. Figure 32 shows the concept of applying a biased AC field.
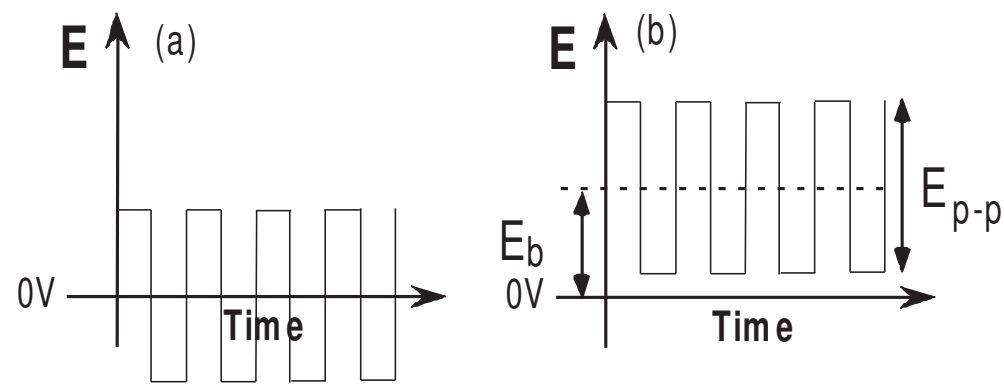

Figure 32. Concept of a biased AC field application. (a) Alternating electric field. (b) Biased alternating electric field.

A typical example of the two-beam coupling signal is observed with the application of a 2.0 $\mathrm{V}_{\mathrm{p}-\mathrm{p}} \mu \mathrm{m}^{-1}, 100 \mathrm{~Hz}$ square waveform electric field without a bias field $\left(\mathrm{E}_{\mathrm{b}}=0\right)$, as shown in Figure 33(a). The transmitted intensities of the laser beams through the FLC/CDH/TNF mixture upon the application of an alternating electric field as a function of time is shown in the figure. The interference of the divided beams in the sample resulted in the increased transmittance of one of the beams and the decreased transmittance of the other. Although the transmitted intensity of the laser beam oscillates due to the switching motion of the FLC molecules, the average intensities of the beams were symmetrically changed, as shown in Figure 33(a). This indicates that a grating based on the spatial difference in the rotational switching motion of FLC molecules was formed and acted as a diffraction grating.
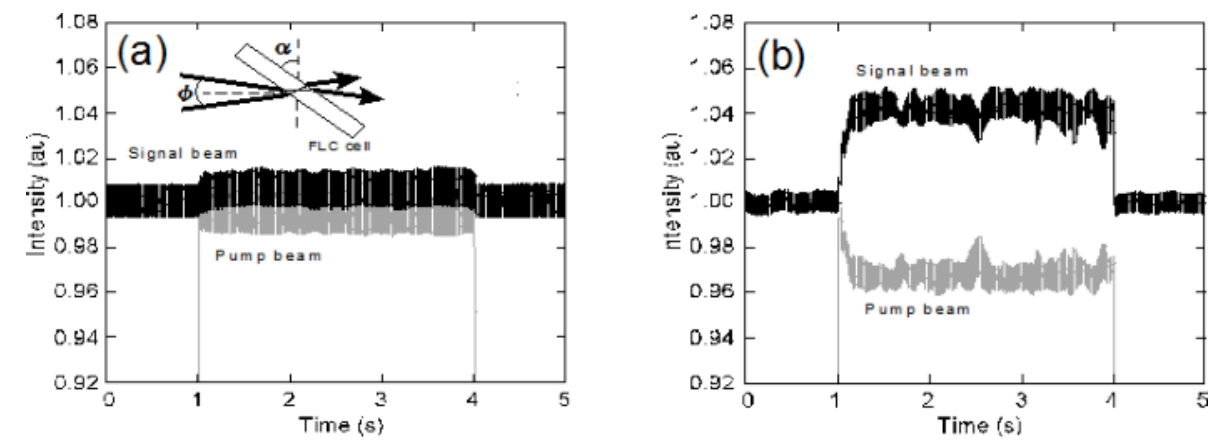

Figure 33. FigAsymmetric energy exchange with a square waveform of $2.0 \mathrm{~V}_{\mathrm{p}-\mathrm{p}} \mu \mathrm{m}^{-1}, 100 \mathrm{~Hz}(\mathrm{a})$ without bias, and (b) with a bias of $0.5 \mathrm{~V} \mathrm{\mu m}^{-1}$. The intersection angle $\phi$ was $20^{\circ}$ and the sample angle $a$ was $50^{\circ}$. 
The asymmetric energy exchange between the signal and the pump beams proves the formation of a diffraction grating phase-shifted from the interference fringe. The grating is considered to be based on the spatial difference in the rotational switching motion of FLC molecules. When the biased electric field was set to $E_{b}=0.5 \mathrm{~V} \mu \mathrm{m}^{-1}$, the two-beam coupling signal was dramatically enhanced (Figure 33(b)). The gain coefficient increased from $10 \mathrm{~cm}^{-1}$ $\left(E_{b}=0\right)$ to $70 \mathrm{~cm}^{-1}\left(E_{b}=0.5 \mathrm{~V} \mathrm{\mu m}{ }^{-1}\right)$. Obviously, the biased field contributes to the formation of the diffraction grating. The internal electric field is thought to be difficult to form under an AC electric field. It has been reported that ionic conduction is the major contributor to the formation of the space-charge field in the photorefractive effect of an FLC. The anisotropic mobility of ionic species in an LC medium affects the formation of the field. In the experimental conditions reported, the interference fringe is formed across the smectic layer so that migration of ionic species occurs in the inter-layer direction. The asymmetric structure of the surface stabilized state of the FLC may lead to the asymmetric mobility of cations and anions. It was considered that the charge separated state is more effectively formed under a biased AC field.

\section{Conclusion}

The reorientational photorefractive effect based on the response of bulk polarization was observed in dye-doped FLC samples. Photorefractivity was observed only in the ferroelectric phase of these samples and the refractive index formation time was found to be shorter than that of nematic LCs. The response time was in the order of ms and was dominated by the formation of the internal electric field. These results indicate that the mechanism responsible for refractive index grating formation in FLCs is different from that for non-ferroelectric materials and that it is clearly related to the ferroelectric properties of the material. The photorefractivity of FLCs was strongly affected by the properties of the FLCs themselves. Besides, with properties such as spontaneous polarization, viscosity and phase transition temperature, the homogeneity of the SS-state was also found to be a major factor. The gain coefficient, refractive index grating formation time (response time) and stability of the two-beam coupling signal were all strongly affected by the homogeneity of the SS-state. Therefore, a highly homogeneous SS-state is necessary to create a photorefractive device. The techniques employed recently in the development of fine LC display panels will be utilized in the future in the fabrication of photorefractive devices.

\section{Acknowledgment}

The authors would like to thank the Japan Science and Technology Agency (JST) S-innovation and the Canon Foundation for support. 


\section{Author details}

Takeo Sasaki

Tokyo University of Science, Japan

\section{References}

[1] Yeh, P. Introduction to Photorefractive Nonlinear Optics; John Wiley: New York, 1993.

[2] Solymar, L.; Webb, J. D.; Grunnet-Jepsen, A. The Physics and Applications of Photorefractive Materials; Oxford: New York, 1996.

[3] Moerner, W. E.; Silence, S. M. Chem. Rev. 1994, 94, 127-155.

[4] Meerholz, K.; Volodin, B. L.; Kippelen, B.; Peyghambarian, N. Nature 1994, 371, 497-500.

[5] Kippelen, B.; Marder, S. R.; Hendrickx, E.; Maldonado, J. L.; Guillemet, G.; Volodin, B. L.; Steele, D. D.; Enami, Y.; Sandalphon; Yao, Y. J.; Wang, J. F.; Röckel, H.; Erskine, L.; Peyghambarian, N. Science 1998, 279, 54-56.

[6] Kippelen, B.; Peyghambarian, N. Advances in Polymer Science, Polymers for Photonics Applications II, Springer, 2002, 87-156.

[7] Ostroverkhova, O.; Moerner, W. E. Chem. Rev., 2004, 104, 3267-3314.

[8] Sasaki, T. Polymer Journal, 2005, 37, 797-812.

[9] Tay, S.; Blanche, P. A.; Voorakaranam, R.; Tunc, A. V.; Lin, W.; Rokutanda, S.; Gu, T.; Flores, D.; Wang, P.; Li, G.; Hilarie, P.; Thomas, J.; Norwood, R. A.; Yamamoto, M.; Peyghambarian, N. Nature, 2008, 451, 694-698.

[10] Blanche, P. A.; Bablumian, A.; Voorakaranam, R.; Christenson, C.; Lin, W.; Gu, T.; Flores, D.; Wang, P.; Hsieh, W. Y.; Kathaperumal, M.; Rachwal, B.; Siddiqui, O.; Thomas, J.; Norwood, R. A.; Yamamoto, M.; Peyghambarian, N. Nature, 2010, 468, 80-83.

[11] Wiederrecht, G. P., Yoon, B. A., Wasielewski, M. R. Adv. Materials 2000, 12, 1533-1536.

[12] Sasaki, T.; Katsuragi, A.; Mochizuki, O.; Nakazawa, Y. J. Phys. Chem. B, 2003, 107, $7659-7665$.

[13] Talarico, M.; Goelemme, A. Nature Mater., 2006, 5, 185-188.

[14] Sasaki, T; Miyazaki, D.; Akaike, K.; Ikegami, M.; Naka, Y. J. Mater. Chem., 2011, 21, 8678-8686. 
[15] Skarp, K.; Handschy, M. A. Mol. Cryst. Liq. Cryst. 1988, 165, 439-569.

[16] Sasaki, T.; Mochizuki, O.; Nakazawa, N.; Fukunaga, G.; Nakamura, T.; Noborio, K. Appl. Phys. Lett., 2004, 85, 1329-1331.

[17] Sasaki, T.; Mochizuki, O.; Nakazawa, Y.; Noborio, K. J. Phys. Chem. B, 2004, 108, 17083-17088. 